\title{
PISTIS: An Event-Triggered Real-Time Byzantine-Resilient Protocol Suite
}

\author{
David Kozhaya $^{1}$, Jérémie Decouchant ${ }^{2, *}$, Vincent Rahli ${ }^{3}$, and Paulo Esteves-Verissimo ${ }^{4, *}$ \\ ${ }^{1}$ ABB Research Switzerland; ${ }^{2}$ TU Delft; ${ }^{3}$ University of Birmingham; ${ }^{4}$ KAUST - RC3
}

\begin{abstract}
The accelerated digitalisation of society along with technological evolution have extended the geographical span of cyber-physical systems. Two main threats have made the reliable and real-time control of these systems challenging: (i) uncertainty in the communication infrastructure induced by scale, and heterogeneity of the environment and devices; and (ii) targeted attacks maliciously worsening the impact of the above-mentioned communication uncertainties, disrupting the correctness of realtime applications.

This paper addresses those challenges by showing how to build distributed protocols that provide both real-time with practical performance, and scalability in the presence of network faults and attacks, in probabilistic synchronous environments. We provide a suite of real-time Byzantine protocols, which we prove correct, starting from a reliable broadcast protocol, called PISTIS, up to atomic broadcast and consensus. This suite simplifies the construction of powerful distributed and decentralized monitoring and control applications, including state-machine replication. Extensive empirical simulations showcase PISTIS's robustness, latency, and scalability. For example, PISTIS can withstand message loss (and delay) rates up to $50 \%$ in systems with 49 nodes and provides bounded delivery latencies in the order of a few milliseconds.
\end{abstract}

Index Terms-real-time distributed systems, probabilistic losses, consensus, atomic broadcast, Byzantine resilience, intrusion tolerance.

\section{INTRODUCTION}

The accelerated digitalisation of society has significantly shifted the way that physical infrastructures-including large continuous process plants, manufacturing shop-floors, power grid installations, and even ecosystems of connected carsare operated nowadays. Technological evolution has made it possible to orchestrate a higher and finer degree of automation, through the proliferation of multiple sensing, computing, and communication devices that monitor and control such infrastructures. These monitoring and control devices are distributed by nature of the geographical separation of the physical processes they are concerned with. The overall systems, i.e., the physical infrastructures with their monitoring and control apparatus, are generally known as cyber-physical systems (CPS) [1]. However, transposing the monitoring and control functionality normally available in classical, real-time (i.e., adhering to given time bounds) and embedded systems, to the distributed CPS scenarios mentioned above, is a very challenging task, due to two main reasons.

First, the scale of the systems as well as the heterogeneity of devices (sensors, actuators and gateways), induce uncertainty in the communication infrastructure interconnecting them, itself often diverse too, e.g., Bluetooth, Wireless IEEE

\footnotetext{
${ }^{*}$ Work partly performed while these authors were with the University of Luxembourg.
}

802.11, or Fiber [2-5]. These communication uncertainties become evident [3-5], namely in the form of link faults and message delays, which hamper the necessary reliability and synchronism needed to realize real-time operations, be it when fetching monitoring data or when pushing decisions to controllers.

Second, security vulnerabilities of many integrated devices, as well as the criticality of the managed physical structures, increase the likelihood of targeted attacks [6, 7]. Such attacks can aim to inflict inconsistencies across system components or to disrupt the timeliness and correctness of real-time applications. The consequences of such attacks can range from loss of availability to severe physical damage [8].

This paper addresses the challenges above, which render traditional approaches for building real-time communications, ineffective in wide-scale, uncertain, and vulnerable settings. We investigate, in particular, how to build large-scale distributed protocols that can provide real-time communication guarantees and can tolerate network faults and attacks, in probabilistic synchronous environments. These protocols simplify the construction of powerful distributed monitoring and control applications, including state-machine replication for fault tolerance. To our knowledge, literature, with the exception of $[9,10]$, has targeted achieving either real-time guarantees or Byzantine-resilience with network uncertainties, but not both.

To bridge this gap, we present a protocol suite of realtime Byzantine protocols, providing several message delivery semantics, from reliable broadcast $\left(P I S T I S^{1}\right)$, through consensus (PISTIS-CS), to atomic broadcast (PISTIS-AT). PISTIS is capable of: (i) delivering real-time practical performance (i.e., correct nodes provide guarantees within given time bounds) in the presence of aggressive faults and attacks (i.e., one third of the nodes being Byzantine, and high message loss rates); and (ii) scaling with increasing system size.

The main idea underlying PISTIS is an event-triggered signature based approach to constantly monitor the network connectivity among processes. Connectivity is measured thanks to the broadcast messages: processes embed signed monitoring information within the messages of the broadcast protocol and exclude themselves from the protocol when they are a threat to timeliness. Hence, PISTIS does not modularly build on membership/failure detector oracles (like in traditional distributed computing) but rather directly incorporates such functionalities within. In fact, modularity in this sense was proven to be impossible for algorithms implementing PISTISlike guarantees [10]. In order to mask network uncertainties

${ }^{1}$ PISTIS was a Greek goddess who represented the personified spirit (daimona) of trust, honesty and good faith. 
in a scalable manner, PISTIS uses a temporal and spatial gossip-style message diffusion with fast signature verification schemes.

We empirically show that PISTIS is robust. For example PISTIS can tolerate message loss rates of up to $40 \%, 50 \%$, $60 \%$, and $70 \%$ in systems with $25,49,73$, and 300 nodes respectively: PISTIS has a negligible probability of being unavailable under such losses. We also show that PISTIS can meet the strict timing constraints of a large class of typical CPS applications, mainly in Supervisory Control And Data Acquisition (SCADA) and Internet of Things (IoT) areas, e.g., (1) fast automatic interactions $(\leq 20 \mathrm{~ms})$ for systems with up to 200 nodes, (2) power systems and substation automation applications $(\leq 100 \mathrm{~ms})$ for systems with up to 1000 nodes, and (3) slow speed auto-control functions $(\leq 500 \mathrm{~ms})$, continuous control applications $(\leq 1 \mathrm{~s})$ as well as operator commands of SCADA applications $(\leq 2 \mathrm{~s})$ for systems with 1000 nodes or more. Such SCADA and IoT applications could include up to hundreds of devices where reliable and timely communication is required.

By using PISTIS as the baseline real-time Byzantine reliable broadcast protocol, we prove that (and show how) higher-level real-time Byzantine resilient abstractions can be modularly implemented, namely, consensus and atomic broadcast. Interestingly, we prove that this can be realized with negligible effort: (1) we exhibit classes of algorithms which are amenable to real-time operations by re-using existing synchronous algorithms from the literature; and (2) we rely on PISTIS, which addresses and tolerates the most relevant problems posed by the communication environment, including the impossibility of modularly handling membership/failure detection [10].

In short, our contributions are:

- The PISTIS protocol suite, which is to the best of our knowledge the first generic and modular protocol suite that provides message delivery guarantees for protocols ranging from Byzantine reliable broadcast to Byzantine atomic broadcast. PISTIS itself is an event-triggered real-time Byzantine reliable broadcast algorithm that has higher scalability and faster message delivery than conventional time-triggered real-time algorithms, in the presence of randomized and unbounded network disruptions. Building on top of PISTIS, we present classes of algorithms, PISTIS-CS and PISTIS-AT, that implement real-time Byzantine consensus and atomic broadcast, respectively.

- Correctness proofs of the PISTIS protocol suite. We provide the main proof results in this paper (exhaustive proofs are deferred to Appx. B).

- Extensive empirical simulations using Omnet++ [11] that showcase PISTIS's robustness, latency, and scalability.

Roadmap. The rest of the paper is organized as follows. Sec. II discusses related work. Sec. III details our system model. Sec. IV recalls the properties of a real-time Byzantine reliable broadcast, and presents our algorithm, PISTIS, in details. Sec. V shows and proves how real-time Byzantine atomic broadcast and consensus can be realized on top of PISTIS's guarantees using classes of existing algorithms. Sec. VI evaluates the performance and reliability of PISTIS. Finally, Sec. VII concludes the paper. For space limitations, proofs and additional material are deferred to Appendices.

\section{RELATED WORK}

Reliable broadcast is a standard abstraction to ensure that the (correct) nodes of a distributed system agree on the delivery of messages even in the presence of faulty nodes. Byzantine reliable broadcast in particular guarantees that (correct) nodes agree even in the presence of arbitrary faults. It is a key building block of reliable distributed systems such as Byzantine Fault-Tolerant State Machine Replication protocols, which are nowadays primarily used in blockchain systems. Pioneered by the work of Dolev [12] and Bracha [13], many protocols have been proposed since then that are intended to work in various environments. The focus of our paper is on novel Byzantine broadcast primitives and protocols that achieve timeliness guarantees.

This paper has evolved from, and improved over, a research line paved by $[9,10,14]$ on timing aspects of reliable broadcast and Byzantine algorithms. Besides these works, the literature on broadcast primitives, to the best of our knowledge, either does not take into account timeliness and maliciousness or addresses them separately.

Cristian et al. [9] assumed that all correct processes remain synchronously connected, regardless of process and network failures. This strong network assumption is too optimistic, both in terms of scale and timing behaviour, which in practice leads to poor performance (latency of approximately 2.4 seconds with 25 processes-see Table I in Sec. VI-E for more details). Moreover, Cristian et al.'s system model does not allow processes that malfunction (e.g., by violating timing assumptions) to know that they are treated as faulty by the model. Our algorithm, in comparison, provides latencies in the range of few milliseconds and our model makes processes aware of their untimeliness.

Verissimo et al. [14] addressed the timeliness problem by weak-fail-silence: despite the capability of the transmission medium to deliver messages reliably and in real-time, the protocol should not be agnostic of potential timing or omission faults (even if sporadic). The bounded omissions assumption (pre-defined maximum number of omissions) of [14] could not be taken as is, if we were to tolerate higher and more uncertain faults (as we consider in this paper): it could easily lead to system unavailability in faulty periods. Hence we operate with much higher uncertainty levels (faults and attacks).

Kozhaya et al. [10] devised a Byzantine-resilient algorithm that provides an upper bound on the delivery latency of messages. This algorithm is time-triggered and relies on an all-to-all communication that limits the algorithm's scalability. Our work improves over [10] on several points: (i) we reduce the delivery latency (few milliseconds as shown in Fig. 7 and Fig. 8 compared to a few hundred as shown in [10, Fig. 8]see also Table I for a comparison of worst case latencies) by adopting an event-triggered approach instead of a roundbased one; (ii) we improve the system's scalability (at least 5 times less bandwidth consumption) by adopting a gossipbased dissemination instead of an all-to-all communication; 
and (iii) we show how real-time broadcast primitives can be modularly used to build real-time Byzantine-resilient highlevel abstractions like consensus and atomic broadcast.

Guerraoui et al. [15] designed a scalable reliable broadcast abstraction that can also be used in a probabilistic setting where each of its properties can be violated with low probability. They achieve a scalable solution by relying on stochastic samples instead of quorums, where samples can be much smaller than quorums. As opposed to this work, our goal is to design a deterministic abstraction where the property are never violated: the real-time Byzantine-resilient reliable broadcast primitive discussed in Sec.IV is deterministic because late processes become passive, and therefore count as being faulty.

In [16, 17], the authors present a Byzantine fault-tolerant SCADA system that relies on the Prime [18, 19] Byzantine Fault Tolerant State Machine Replication [20, 21] (BFT-SMR) protocol protocol to ensure both safety and latency guarantees. As opposed to PISTIS, Prime relies on an asynchronous primary-based BFT-SMR. As opposed to Prime, PISTIS-CS and PISTIS-AT algorithms are designed modularly from a timely reliable broadcast primitive; and PISTIS allows slow connections between any processes in a probabilistic synchronous environment, while Prime relies on the existence of a "stable" timely set of processes.

\section{SySTEM AND THREAT MODEL}

\section{A. System Model}

Processes. We consider a distributed system consisting of a set $\Pi=\left\{p_{0}, p_{1}, \ldots, p_{N-1}\right\}$ of $N>1$ processes. We assume that processes are uniquely identifiable and can use digital signatures to verify the authenticity of messages and enforce their integrity. We denote by $\sigma_{i}(v)$ the signature of value $v$ by process $p_{i}$. We often write $\sigma_{i}$, when the payload is clear from the context. Processes are synchronous, i.e., the delay for performing a local step has a fixed known bound (note that this does not apply to faulty processes-see below).

Clocks. Processes have access to local clocks with a bounded and negligible rate drift to real time. These clocks do not need to be synchronized.

Communication. Every pair of processes is connected by two logical uni-directional links, e.g., $p_{i}$ and $p_{j}$ are connected by links $l_{i j}$ and $l_{j i}$. Links can abstract a physical bus or a dedicated network link. We assume a probabilistic synchronous communication model. This means that in any transmission attempt to send a message over on link $l_{i j}$ (with $i \neq j$ ) at some time $t$, there is a probability $P_{i j}(t)$ that the message reaches its destination and within a maximum delay $d$ (known to the processes). $d$ is the upper time bound on non-lossy message delivery and $\epsilon_{1}<1-P_{i j}(t)<\epsilon_{2} \ll 1$ where $\epsilon_{1}$ and $\epsilon_{2}$ are small strictly positive values. Such violations exist in networks, as arguably all communication is prone to unpredictable disturbances, e.g., bandwidth limitation, bad channel quality, interference, collisions, and stack overflows [4]. Our probabilistic synchronous communication has been shown to be weaker, in some sense [22], than partial synchrony [23]. We further discuss and compare our model to existing traditional ones in Appx. A. We do not model correlated losses explicitly, as previous works like [10] have shown that such bursts can be mitigated and we leave it up to the applications to define how to deal with late messages (i.e., violating the $d$ delay assumption).

\section{B. Threat Model}

Processes. We assume that some processes can exhibit arbitrary, a.k.a. Byzantine, behavior. Byzantine nodes can abstract processes that have been compromised by attackers, or are executing the algorithm incorrectly, e.g., as a result of some fault (software or hardware). A Byzantine process can behave arbitrarily, e.g., it may crash, fail to send or receive messages, delay messages, send arbitrary messages, etc.

We assume that at most $f=\left\lfloor\frac{N-1}{3}\right\rfloor$ processes can be Byzantine. This formula was proved to be an upper bound for solving many forms of agreement in a variety of models such as in non-synchronous models [24, 25].

We allow nodes to become passive in case they fail to execute in a timely fashion. As explained in Sec. IV-C, passive nodes stop executing key events to guarantee timeliness. A process that exhibits a Byzantine behavior or that enters the passive mode (see Sec. IV-C) is termed faulty. Otherwise, the process is said to be correct. Note that passive nodes are considered faulty (at least) during the time they are passive, but are not counted against the $f$ Byzantine faults. Therefore, more than $f$ nodes could be faulty in a system over the full lifespan of a system (up to $f$ nodes could be Byzantine, and up to $N$ processes could be momentarily passive).

Clocks. The bounded and negligible rate drift assumption in Sec. III-A has to hold only on a per protocol execution basis, easily met by current technology (such as techniques relying on GPS [26] or trusted components [27]). Hence the clock of a correct process always behaves as described in Sec. III-A.

Communication. We assume that Byzantine processes or network adversaries cannot modify the content of messages sent on a link connecting correct processes (implemented by authentication through unforgeable signatures [28]).

\section{REAL-Time ByZANTINE RELIABLE BROADCAST}

We now present our solution to guarantee that correct nodes reliably deliver broadcast messages in a timely fashion, despite Byzantine nodes, and communication disruptions. Sec. IV-A recalls the properties of the real-time Byzantine-resilient reliable broadcast (RTBRB) primitive [10]. Then, Sec. IV-B presents a high-level overview of the PISTIS event-triggered algorithm, which implements the RTBRB primitive, while Sec. IV-C provides a detailed presentation of PISTIS. Finally, Sec. IV-E explains how passive nodes can recover and become active again to ensure the liveness of the system.

\section{A. Real-time Byzantine Reliable Broadcast Abstraction}

Definition 1 (RTBRB). The real-time Byzantine reliable broadcast $(R T B R B)$ primitive guarantees the following properties [10], assuming every message is uniquely identified (e.g., 
using the pair of a sequence number and a process id-the broadcaster's id). ${ }^{2}$ In this abstraction, a process broadcasts a message by invoking RTBRB-broadcast(). Similarly, a process delivers a message by invoking $R T B R B-d e I i$ ver().

- RTBRB-Validity: If a correct process p broadcasts $m$, then some correct process eventually delivers $m$.

- RTBRB-No duplication: No correct process delivers message $m$ more than once.

- RTBRB-Integrity: If some correct process delivers a message $m$ with sender $p_{i}$ and process $p_{i}$ is correct, then $m$ was previously broadcast by $p_{i}$.

- RTBRB-Agreement: If some correct process delivers m, then every correct process eventually delivers $m$.

- RTBRB-Timeliness: There exists a known $\Delta_{\mathrm{R}}$ such that if a correct process broadcasts $m$ at real-time $t$, no correct process delivers $m$ after real time $t+\Delta_{\mathrm{R}}$.

It is important to note that the above abstraction does not enforce ordering on the delivery of messages sent. We elaborate more on that and how to achieve order in Sec. V. Note also that in a system consisting of correct and faulty nodes, these properties ensure that correct nodes deliver broadcast messages within a bounded delay, while no such guarantee is (and can be) provided about faulty nodes.

\section{B. Overview of PISTIS}

This section presents a high-level description of PISTIS. For simplicity, we assume the total number of processes to be $N=3 f+1$, in which case a Byzantine quorum has a size of $2 f+1$. PISTIS guarantees RTBRB properties deterministically despite the probabilistic lossy network. However, this comes at the price of PISTIS triggering an entire system failsafe (shutdown) and a reinitialization of system state when violating RTBRB-Timeliness is inevitable. We show later in Sec. VI that the probability of PISTIS causing such system fail-safe (and hence violating an RTBRB property if fail-safe was not triggered) is negligible.

System Awareness. Given that broadcasts can be invoked at unknown times, there might exist a correct process in $\Pi \backslash\left\{p_{i}\right\}$ that is unaware of $p_{i}$ 's broadcast for an unbounded amount of time after it was issued, since all links can lose an unbounded number of messages. The occurrence of such scenarios may hinder the system's ability of delivering realtime guarantees. To this end, we require that every process $p_{j}$ constantly exchanges messages with the rest of the system. This regular message exchange aims at capturing how well $p_{j}$ is connected to other processes, and hence to what extent $p_{j}$ is up-to-date with what is going on in the system (and to what extent the system knows about $p_{j}$ 's state). We achieve this constant periodic message exchange via a function, which we call proof-of-connectivity. ${ }^{3}$ It requires each process to diffuse heartbeats to the rest of the system in overlapping rounds: a

\footnotetext{
${ }^{2}$ RTBRB's properties are equivalent to the ones of the Byzantine reliable broadcast abstraction defined in [29, Module 3.12, p.117], excluding Timeliness.

${ }^{3}$ Periodic message exchange (heartbeats) has been used to discover the network state in many monitoring algorithms [30, 31]
}

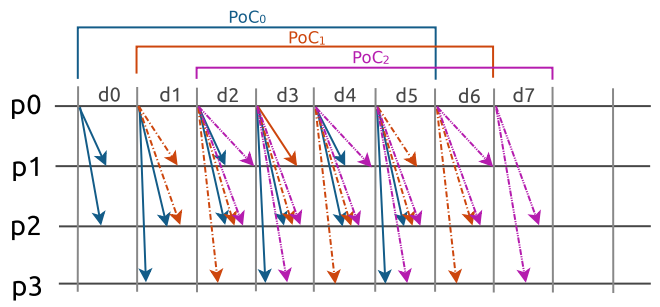

Figure 1. Example of a proof-of-connectivity run, where $X=2 f+1$, and where 2 repetitions allow covering all nodes

new round is started every $d$ time units, and each round is of a fixed duration $\mathbb{T}$, where $d<\mathbb{T}$. (Sec. VI shows that $\mathbb{T}=8 d$ is a reasonably good value, while Sec. IV-D highlights the need for overlapping rounds.) A round consists in repeatedly (every $d$ units of time) diffusing a signed heartbeat message to $X$ other processes. $X$ stands for the number of processes to which a process sends a message in a communication step. The value of $X$ is fixed at deployment time (i.e., does not change over the execution of a system) and can range between 0 and $N-1$. It is used to avoid network congestions by enforcing that processes selectively send their messages to an arbitrary subset of the system. Each round consists then in repeatedly sending $\left\lceil\frac{\mathbb{T}}{d}\right\rceil$ times a message, each time to $X$ other nodes. Note that even though the value of $X$ is fixed, in any given round the set of $X$ processes to which the message is sent in every repetition can change such that the union of processes to which the message is sent in all $\left\lceil\frac{\mathbb{T}}{d}\right\rceil$ repetitions in that round covers all processes in the system. This is possible when $N \leq X \times\left\lceil\frac{\mathbb{T}}{d}\right\rceil$, which we always guarantee in practice. Heartbeat messages are uniquely identified by sequence numbers, which are incremented prior to each round. On receipt of a heartbeat message, a correct process appends its own signature to it as well as all other seen signatures relative to that heartbeat; and sends it to $X$ other processes. At the end of each round, if a process does not receive at least $2 f+1$ signatures (including its own) on its own heartbeat, it enters the passive mode.

Fig. 1 provides an example of a run of the proof-ofconnectivity protocol, depicted as a message sequence diagram, in a system composed of 4 processes. This figure depicts part of the three first rounds of proof-of-connectivity initiated by $p_{0}$ (we only show the messages sent by $p_{0}$ to avoid cluttering the picture), namely $P_{o} C_{0}$ in blue, $P_{o} C_{1}$ in orange, and $\mathrm{PoC}_{2}$ in purple. In addition, in that case, each proof of connectivity round is of length $\mathbb{T}=6 d$. Therefore, the blue $P_{o} C_{0}$ heartbeats are sent 6 times between $d_{0}$ and $d_{5}$, the orange $P_{o} C_{1}$ heartbeats are sent 6 times between $d_{1}$ and $d_{6}$, and the purple $\mathrm{PoC}_{2}$ heartbeats are sent 6 times between $d_{2}$ and $d_{7}$. If by the end of $P_{o} C_{0}, p_{0}$ has not received $2 f$ replies to its heartbeats, it will become passive.

Diffusing Broadcasts. PISTIS relies on two types of messages (Echo and Deliver messages) to ensure that broadcast values are delivered in a timely fashion. Processes exchange Echo messages either to start broadcasting new values, or in response to received Echo messages. Echo messages help processes gather a valid quorum (a Byzantine write quorum [32] of size $2 f+1$ ) of signatures on a single 


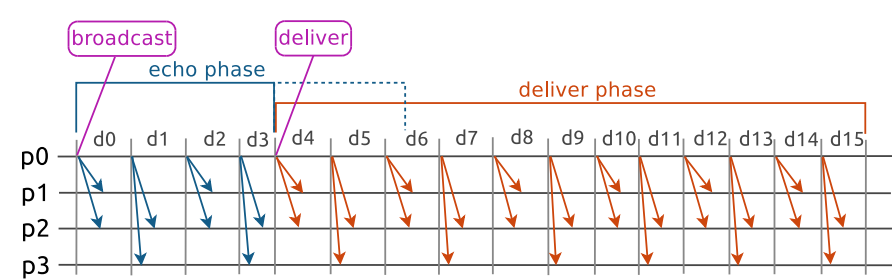

Figure 2. Example of a PISTIS run where $X=2 f+1$, and where 2 repetitions allow covering all nodes

value $v$ relative to a broadcast instance. A broadcast instance is identified by the id of the process broadcasting $v$ and a sequence number. Echo messages help prevent system inconsistencies when malicious nodes send different values with the same sequence number (same broadcast instance) to different recipients. However, additional messages, namely Deliver messages, are needed to help achieve delivery within a bounded time after the broadcast.

When a process $p_{i}$ receives a value $v$ through an Echo message, it appends its signature to the message as well as all other signatures it has received relative to $v$; and sends it to $X$ other processes. In addition, when $p_{i}$ receives a value for the first time, it triggers a local timer of duration $\mathbb{T}$. Upon receiving a value signed by more than $2 f$ processes, a process delivers that value. However, a process that does not receive more than $2 f$ signatures on time (i.e., before the timer expires) enters the passive mode. In case multiple values are heard relative to a single process and sequence number (equivocation), then the first heard value is the one to be echoed. Note that processes continue executing the proofof-connectivity function during the echo and deliver phases however by piggybacking heartbeats to echo/deliver messages.

As opposed to Echo messages that are diffused (i.e., retransmitted temporally and sporadically) for a duration $\mathbb{T}$, Deliver messages are diffused for $2 \mathbb{T}$. This is needed to ensure that if some correct processes start diffusing a message between some time $t$ and $t+\mathbb{T}$, possibly at different times, then there must be a $\mathbb{T}$-long period of time where all of them are diffusing the message (see Lemma 4 in Appx. B for more details). Given a large enough collection of such processes ( $f+1$ correct processes $)$, this allows other processes to learn about delivered values in a timely fashion.

Fig. 2 provides an example of a run of PISTIS, depicted as a message sequence diagram. The system is composed of 4 processes. This figure depicts part of the echo (in blue) and deliver (in orange) phases of one broadcast initiated by $p_{0}$ (for the purpose of this illustration, only the messages sent by $p_{0}$ are shown). The purple "broadcast" and "deliver" tags indicate the times at which $p_{0}$ initiated its broadcast, and delivered it. In this example, the echo phase is initially meant to last for a duration of $\mathbb{T}=6 d$. However, it happens here that $p_{0}$ received $2 f$ echo messages for its broadcast by $3 d+k$, where $0<k<d$, which is why $d_{3}$ is shorter than the other intervals. Therefore, $p_{0}$ stops its echo phase and starts its deliver phase at $3 d+k$. As mentioned above, the deliver phase lasts for $2 \mathbb{T}$. If $p_{0}$ has not received $2 f$ deliver messages in return by the end of that deliver phase, then it becomes passive.

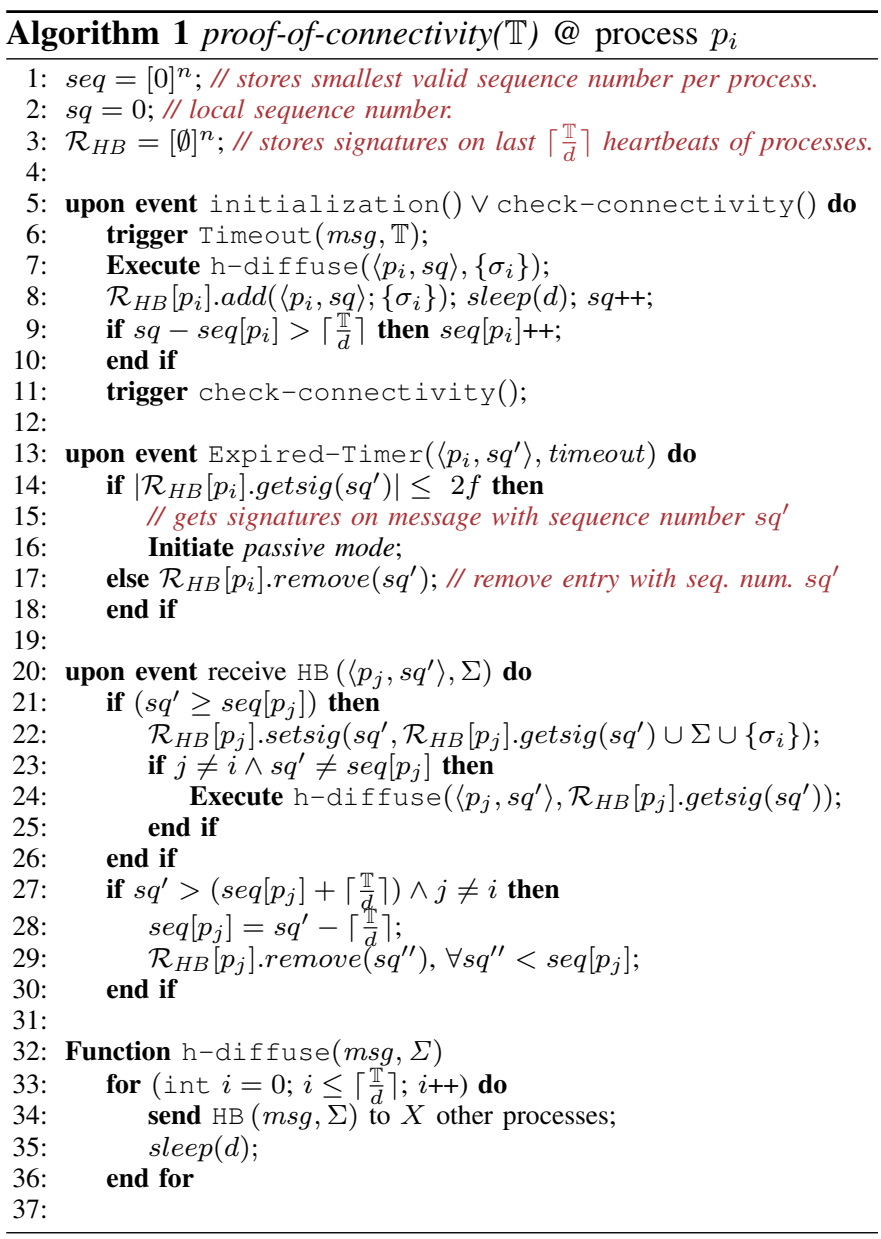

\section{Detailed Presentation of PISTIS}

We now discuss PISTIS (Algorithm 2) in more details. Note that all functions presented in Algorithms 1 and 2 are non-blocking.PISTIS's proof of correctness can be found in Appx. B.

Process states. Processes can become passive under certain scenarios by calling "Initiate passive mode". A passive node stops broadcasting and delivering messages to guarantee timeliness but otherwise keeps on replying to messages to help other processes. Processes that were behaving correctly thus far, are considered faulty when they initiate a passive mode and can notify the application above of this fact. Later in this section, we show how processes in the passive mode can come back to normal operation by calling "Initiate active mode".

Ensuring sufficient connectivity. In PISTIS every process executes the proof-of-connectivity Algorithm 1. Namely, a process $p_{i}$ forms a heartbeat $\mathrm{HB}\left(\left\langle p_{i}, s q\right\rangle,\left\{\sigma_{i}\right\}\right)$, where $s q$ is $p_{i}$ 's current heartbeat sequence number and $\sigma_{i}$ is $p_{i}$ 's signature on $\left\langle p_{i}, s q\right\rangle$. Process $p_{i}$ also stores (in array $\mathcal{R}_{H B}$ ) for every process (including itself) all signatures it receives on heartbeats with a valid sequence number. A valid heartbeat sequence number for some process $p_{j}$ is a sequence number $\geq \operatorname{seq}\left[p_{j}\right]$. Heartbeats with lower sequence numbers are simply ignored. To avoid receiving heartbeats from older rounds, we update $\operatorname{seq}\left[p_{j}\right]$ every time a heartbeat with a sequence number over seq $\left[p_{j}\right]+\left\lceil\frac{\mathbb{T}}{d}\right\rceil$ is receiver (lines 27-28). After 


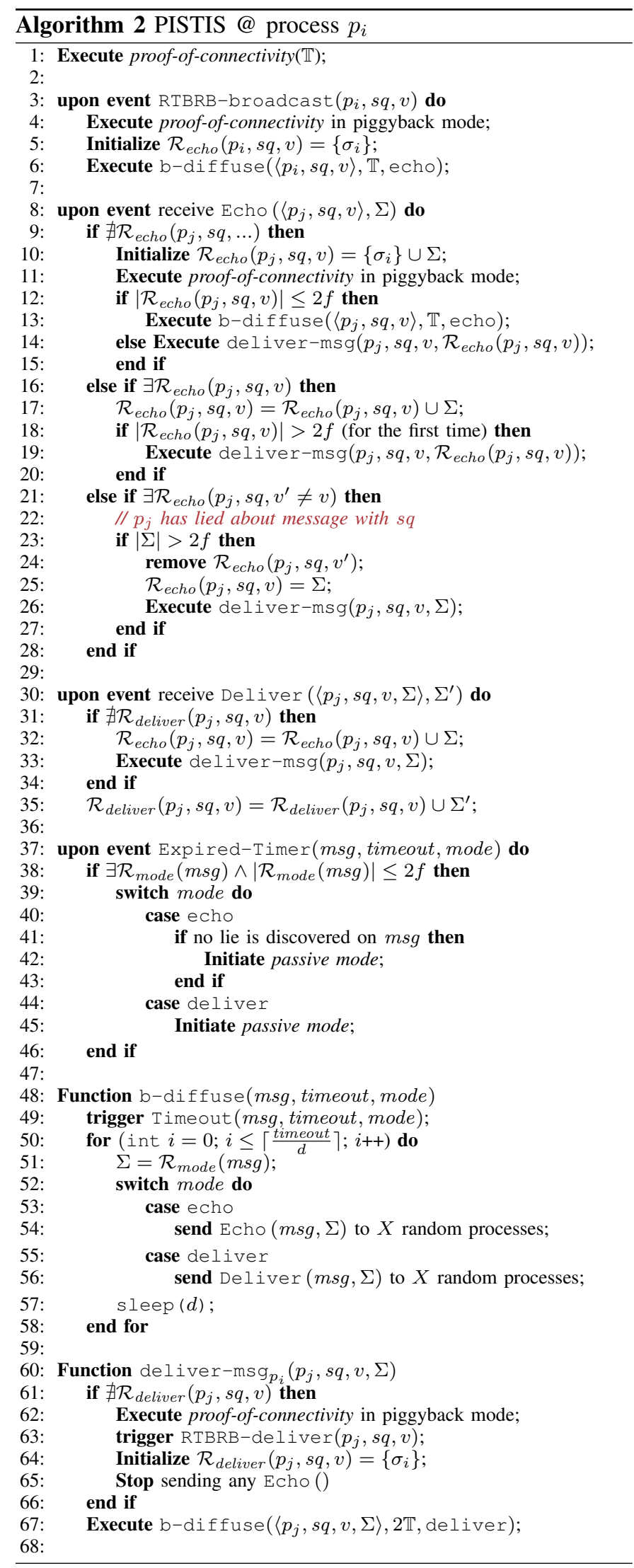


Remark 2. We assume that processes sign payloads of the form $\left(p_{i}, s q, v, \mathrm{E}\right)$ for echo messages and of the form $\left(p_{i}, s q, v, \mathrm{D}\right)$ for deliver messages. We use the $\mathrm{E}$ and $\mathrm{D}$ tags to distinguish echo and deliver payloads, thereby ensuring that an attacker cannot use echo signatures as deliver signatures. Note that echo signatures are sent as part of deliver messages as a proof that a quorum of processes echoed a certain value.

\section{PISTIS' properties}

As mentioned at the beginning of this section, PISTIS is correct in the sense that it satisfies all five properties of the RTBRB primitive presented in Sec. IV-A:

Theorem 1 (Correctness of PISTIS). Under the model presented in Sec. III, the PISTIS algorithm presented in Fig. 2 implements the RTBRB primitive.

A proof of this theorem can be found in Appx. B. Let us point out here that the $\Delta_{R}$ bound of the RTBRB-Timeliness property turns out to be $3 \mathbb{T}$.

Let us also highlight the crux of this proof here.

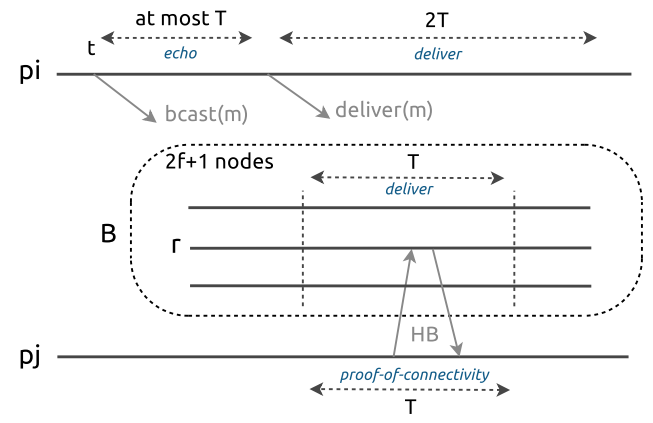

As illustrated above, a correct node $p_{i}$ that broadcasts a message $m$ a time $t$ is guaranteed to start delivering $m$ by $t_{d}=t+\mathbb{T}$. In addition thanks to the $2 \mathbb{T}$ delivery period, we are also guaranteed that a collection, called $B$, of $2 f+1$ nodes, will only deliver $m$ for a $\mathbb{T}$-long period that starts before $t_{d}+\mathbb{T}$. PISTIS's proof-of-connectivity (PoC) mechanism then ensures that any other correct node $p_{j}$ will execute a PoC round during which a correct node $r \in B$ delivers $m$ to $p_{j}$, piggybacked to a heartbeat, thereby guaranteeing that $p_{j}$ delivers $m$ timely.

In particular, overlapping $\mathrm{PoC}$ rounds allow for all correct nodes to have a $\mathrm{PoC}$ round that coincide with that $\mathbb{T}$-long period (called $D$ here), during which the correct nodes in $B$ deliver $m$, thereby allowing all correct nodes to deliver $m$. If PoC rounds were consecutive and not overlapping, a correct node could miss the deliver message (piggybacked with PoC messages) sent during $D$ if it were to receive PoC messages for a round (i.e., sequence number) $s$ sent before $D$, and for round $s+1$ sent after $D$, thereby staying active while not delivering.

\section{E. Byzantine-Resilient Recovery}

If process $p_{i}$ detects that it is executing under bad network conditions, it enters the passive mode and signals the upper application. As a result, $p_{i}$ stops broadcasting and delivering broadcast messages (by not executing line 3 and line 63) to avoid violating RTBRB-Timelines. However, $p_{i}$ continues participating in the dissemination of the broadcast and proofof-connectivity messages to avoid having too many nodes not collecting enough messages and hence becoming passive.

Once the network conditions are acceptable again, $p_{i}$ can recover and resume delivering broadcast messages. More precisely, a process $p_{i}$ that enters passive mode at time $t$ can operate normally again if the interval $\left[t, t+\Delta_{R}\right]$ is free of any passive mode initiations. This $\Delta_{R}$ duration ensures that the messages delivered by a recovered process $p_{i}$ do not violate any RTBRB properties. After a delay $\Delta_{R}$, nodes will resume their full participation in the protocol, and either deliver messages or stay on hold.

Note that in case of multiple broadcast instances, passive nodes that become active again should learn the latest sequence number of broadcasts for other nodes. Otherwise Byzantine nodes can exploit this to hinder the liveness of the system.

Remark 3. Given that processes can now shift between passive and active modes, we specify our notion of correct processes as follows. A system run is modeled by a trace of events happening during that run. An event has a timestamp and a node associated with it. Moreover, an event can either be a correct event or a Byzantine event. Given an algorithm A, a process $p$ is deemed correct w.r.t. A and a trace $\tau$, if: (1) it follows its specification from $e_{1}$, the first correct $A$ related event (i.e., an event of algorithm A) happening in $\tau$, to $e_{2}$, the last correct A-related event happening in $\tau$; (2) p's events between $e_{1}$ and $e_{2}$ must all be correct; (3) p must also have followed its specification since it last started; and (4) $p$ must never have lost its keys (so that no other node can impersonate $p$ when $p$ follows its specification). The results presented below also hold for this definition of correctness, because correct processes are required to be active through the entire broadcast instance.

This recovery mechanism improves the overall resilience of the system. Indeed, having all processes in passive mode can occur if $2 f+1$ nodes are passive, which is now harder to achieve if nodes can recover sufficiently fast enough.

\section{BEYOND A RELIABLE BROADCAST}

Unlike liveness in asynchronous reliable broadcast, the RTBRB-Timeliness property (a safety property) introduces a scent of physical ordering. This ordering is due to the fact that timeliness stipulates, for each execution, a termination event to occur "at or before" some $\Delta_{R}$ on the time-line. This said, the reader may wonder to what extent does the real-time Byzantine-resilient reliable broadcast (of Sec. IV-A) help in establishing total order?

The answer to this question lies in examining what happens to multiple broadcasts issued by the same or by different nodes. When multiple broadcasts interleave, e.g., when they are issued within a period shorter than $\Delta_{R}$ (the upper time bound on delivering a message), messages might be delivered to different processes in different orders. The timeliness property of the real-time Byzantine-resilient reliable broadcast only ensures that a message $m$ that is broadcast at time $t$ is delivered at any time in $\left[t, t+\Delta_{\mathrm{R}}\right]$. Thus, to ensure total order 
on all system events, e.g., for implementing State Machine Replication, additional abstractions need to be built on top of the real-time Byzantine-resilient reliable broadcast primitive that we have developed so far.

In this section, we investigate how to modularly obtain such an order on system events while still preserving realtime and Byzantine-resilience. We define two build blocks that build on top of RTBRB, namely the RTBC real-time Byzantine consensus abstraction (Def. 2) - a fundamental building block for state machine replication, atomic broadcast and leader election [29]; and the RTBAB real-time atomic broadcast abstraction (Def. 4) - to establish total order on system events. We then provide characterizations of classes of algorithms that implement these abstractions: Thm. 2 provides a characterization of the PISTIS-CS class of algorithms that implement RTBC, while Thm. 3 provides a characterization of the PISTIS-AT class of algorithms that implement RTBAB. Finally, we provided examples of algorithms that belong to these classes (see Examples 1 and 2).

We start with the following assumption that constrains the ways processes can communicate.

Assumption 1. Correct processes access the network only via the RTBRB primitive, namely using the two operations: RTBRB-broadcast() and RTBRB-deliver().

From Assumption 1, a correct process $p_{i}$ that receives a message from an operation other than RTBRB-deliver() simply ignores that message by dropping it.

\section{A. Real-Time Byzantine Consensus}

Roughly speaking, solving the Byzantine consensus problem requires the agreement of distributed processes on a given value, even though some of the processes may fail arbitrarily. Byzantine consensus was first identified by Pease et al. [33], and formalized as the interactive consistency problem. An algorithm achieves interactive consistency if it allows the non-faulty processes to come to a consistent view of the initial values of all the processes, including the faulty ones. Once interactive consistency has been reached, the non-faulty processes can reach consensus by applying a deterministic averaging or filtering function on the values of their view. We apply the following assumption to reach consensus.

Assumption 2. Once interactive consistency terminates, every correct process scans the obtained vector and decides on the value that appears at least $2 f+1$ times. If no such value exists, then the process decides $\perp$, a distinguished element that indicates that no value has been decided.

Definition 2 (RTBC). The real-time Byzantine consensus $(R T B C)$ abstraction is expressed by the following properties: ${ }^{4}$

- $\boldsymbol{R T B C}$-Validity: If all correct processes propose the same value $v$, then any correct process that decides, decides $v$. Otherwise, a correct process may only decide a value that was proposed by some correct process or $\perp$.

\footnotetext{
${ }^{4}$ The properties of RTBC are the same as the ones of the traditional (strong) Byzantine consensus defined in [23] (see also [29, Module 5.11, p.246]), excluding the Timeliness property.
}

- RTBC-Agreement: No two correct processes decide differently.

- RTBC-Termination: Correct processes eventually decide.

- RTBC-Timeliness: If a correct process $p_{i}$ proposes a value to consensus at time $t$, then no correct process decides after $t+\Delta_{\mathrm{C}}$.

In RTBC a process $p_{i}$ can propose a value $v$ to consensus by invoking $\mathrm{RTBC}-\mathrm{propose}\left(p_{i}\right.$, inst, $\left.v\right)$, where inst is a sequence number that uniquely identifies a RTBC instance. Similarly, a process $p_{i}$ decides on a value $v$ by invoking $\mathrm{RTBC}$-decide $\left(p_{i}\right.$, inst, $\left.v\right)$. In addition $\mathrm{RTBC}-$ init(inst) instantiate a new instance of RTBC with id inst, i.e., for sequence number inst.

Definition 3. An algorithm is said to be bounded if it only uses a known bounded number of communication rounds.

Theorem 2 (Characterization of the PISTIS-CS class). Let PISTIS-CS be the class of bounded (Def. 3) algorithms that implements interactive consistency under Assumptions 1 and 2. Then, PISTIS-CS algorithms also implement RTBC in our model (described in Sec. III).

See Appx. C for a proof of this result.

Example 1 (Examples of PISTIS-CS algorithms). Because the interactive consistency problem has been solved using different algorithms that satisfy Def. 3, our result applies to various existing algorithms, such as [33-36].

\section{B. Real-Time Byzantine-Resilient Atomic Broadcast}

Definition 4 (RTBAB). A real-time Byzantine-resilient atomic broadcast (RTBAB) has the same properties as RTBRB (with a different timeliness bound) plus an additional ordering property (therefore, we only present the properties that differ from RTBRB's):

- RTBAB-Timeliness: There exists a known $\Delta_{\mathrm{A}}$ such that if a correct process broadcasts $m$ at time $t$, no correct process delivers $m$ after real time $t+\Delta_{\mathrm{A}}$.

- RTBAB-Total order: Let $m_{1}$ and $m_{2}$ be any two messages and suppose that $p_{i}$ and $p_{j}$ are any two correct processes that deliver $m_{1}$ and $m_{2}$. If $p_{i}$ delivers $m_{1}$ before $m_{2}$, then $p_{j}$ delivers $m_{1}$ before $m_{2}$

We now define the class of algorithms (called $\mathcal{R}$ oundBased), through the properties listed below, that modularly implement RTBAB properties. R RoundB ased algorithms make use of a single RTBRB instance and multiple instances of RTBC. We first constrain a $\mathcal{R}$ oundB ased algorithm to start an RTBRB instance within a bounded amount of time for any broadcast call.

Property 1. If a correct process $p_{i}$ RTBAB-broadcasts a message $m$ at time $t$, then it also RTBRB-broadcasts $m$ by time $t+\Delta_{\mathrm{B}}$, for some bounded $\Delta_{\mathrm{B}}$.

We then require a $\mathcal{R}$ ound $\mathcal{B}$ ased algorithm to start (or end in case this has already been done before) an RTBC instance, within a bounded amount of time, every time the RTBRB instance delivers. 
Property 2. If a correct process RTBRB-delivers a message $m$ at time $t$, such that $m$ 's broadcaster is also correct, then it either RTBC-proposes or RTBC-decides $m$ by $t+\Delta_{\mathrm{P}}$, for some bounded $\Delta_{\mathrm{P}}$.

In addition, the next property constrains the values that can be proposed at each RTBC instance, namely that at most one non- $\perp$ value can be proposed at each instance.

Property 3. Given an RTBC instance inst, there exists a value $v$, such that each correct process either RTBC-propose $v$ or $\perp$ at inst.

Next, we require a $\mathcal{R}$ ound $\mathcal{B}$ ased algorithm to deliver a RTBC-decided value within a bounded amount of time (Property 4) and to ensure that non-RTBC-decided values are reproposed in later RTBC rounds (Property 5).

Property 4. If a correct process $R T B C$-decides a message $m$ at time $t$, then it also RTBAB-delivers $m$ by time $t+\Delta_{\mathrm{D}}$, for some bounded $\Delta_{D}$.

Property 5. A correct process $p_{i}$ that proposes a value $v$ at a given time $t$, using a given RTBC instance inst, and such that this instance does not decide $v$, also RTBC-propose $v$ at some instance inst $+k$, where $0<k$. Moreover, $p_{i} R T B C$-proposes $v$ at the smallest instance between inst +1 and inst $+k$ where $m$ is proposed by some process.

Finally, we require that nodes participate in all successive $\mathrm{RTBC}$ instances in a monotonic fashion.

Property 6. Correct processes RTBC-propose exactly one value per RTBC instance; propose values in all RTBC instances (i.e., for all instances inst $\in \mathbb{N}$ ); in increasing order w.r.t. the instance numbers of the RTBC instances (i.e., if $p_{i}$ proposes values at times $t_{1}$ and $t_{2}$ using the $R T B C$ ${\text { instances } \text { inst }_{1} \text { and inst }}_{2}$, respectively, and $t_{1}<t_{2}$, then inst $_{1}<$ inst $_{2}$ ); and not in parallel (i.e., if $p_{i}$ proposes a value at time $t$ using an RTBC instance inst, and that this $R T B C$ instance has not decided by time $t^{\prime}>t$, then $p_{i}$ does not propose any other value between $t$ and $t^{\prime}$ ).

Definition 5. Let $\mathcal{R}$ oundB $\mathcal{B}$ ased be the class of round-based algorithms that satisfy the properties 1, 2, 3, 4, 5, and 6.

Theorem 3 (Characterization of the PISTIS-AT class). Let PISTIS-AT be the class of $\mathcal{R}$ oundB $\mathcal{B}$ ased algorithms that implement the traditional Byzantine total-order broadcast under Assumption 1. Then, PISTIS-AT algorithms also implement $R T B A B$ in our system (described in Sec. III).

To prove Theorem 3, it is sufficient to prove that a RTBABbroadcasted value $m$ is always RTBAB-delivered within a bounded amount of time. Because of the round-based property, $m$ must be RTBRB-proposed and RTBRB-decided within a bounded amount of time. Consequently there is (within a bounded amount of time) an RTBC instance where "enough" correct nodes RTBC-propose $m$, so that $m$ gets RTBC-decided upon and RTBAB-delivered within a bounded amount of time. The proof of Theorem 3 is detailed in Appx. D.

We have introduced bounds for each of the operations executing in bounded time, namely $\Delta_{R}$ (Def. 1), $\Delta_{C}$ (Def. 2),

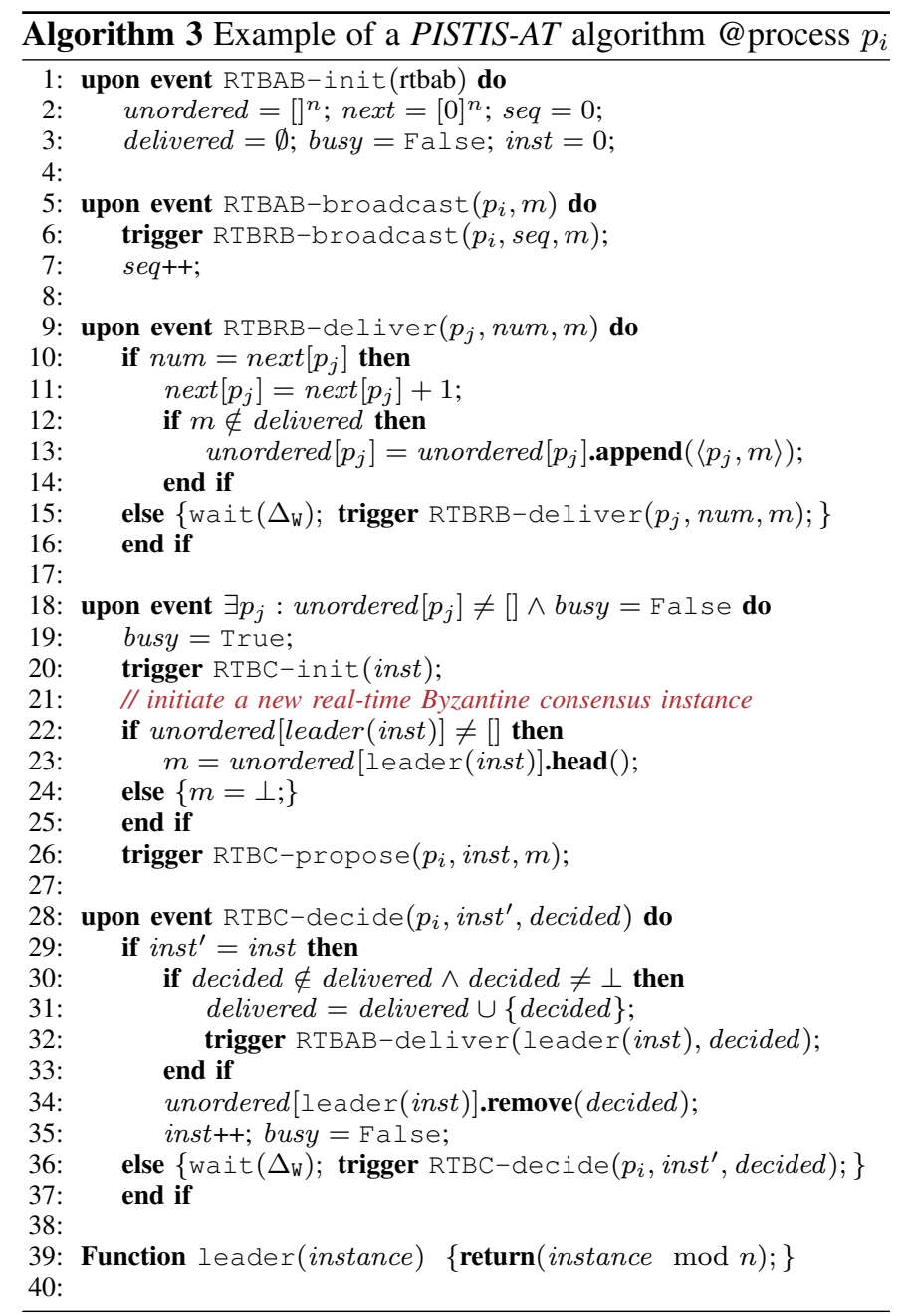

$\Delta_{\mathrm{W}}$ (Alg. 3), $\Delta_{\mathrm{B}}$ (Prop. 1), $\Delta_{\mathrm{P}}$ (Prop. 2), $\Delta_{\mathrm{D}}$ (Prop. 4), and $\Delta_{\mathrm{A}}$ (Def. 4). Those bounds are not assumed to be related to each other. However, the bound for $\Delta_{A}$ we exhibit in Theorem 3's proof is a combination of all the other bounds discussed above.

Example 2 (Example of a PISTIS-AT algorithm). Finally, algorithm 3 provides an example of a PISTIS-AT algorithm that implements RTBAB modularly, which we adapted from [29, Alg.6.2, p.290] to guarantee timeliness.

\section{EVALUATION AND COMPARISON}

In this section, we evaluate PISTIS's reliability, latency, and incurred overhead on network bandwidth.

\section{A. PISTIS's latency vs. related systems' latency}

We begin with a latency comparison between PISTIS and other related works based on the worst case incurred delay. We compute worst case delays from the bounds established for each algorithm (a direct experimental evaluation would not be fair, since not all previous work [9] consider probabilistic synchronous networks). Later sections provide an experimental comparison with RT-ByzCast [10], the system most related to ours. We elaborate in what follows on the computation of the worst case delays. First we refine the definition of $d$ 
introduced in Sec. III-A. Let $d_{n}$ be the maximum network delay, and $d_{p}$ be the maximum local processing time, which includes the cryptographic operations overhead, such that $d$ can be decomposed as $d_{p}+d_{n}$. Christian et al. [9] compute the worst case delay as $10 *(f+2) *(n-1) * d_{n}$ where $f$ is the maximum number of faulty processes, $n$ the total number of processes, and $d_{n}$ the network delay. In this work, $d_{p}$ is equal to 10. Kozhaya et. al [10] compute the worst-case delay as $3 * R * d$, where $R$ is the number of consecutive synchronous communication rounds the same message gets disseminated (time-triggered re-transmissions). PISTIS's worst case delay is proved to be $3 * \mathbb{T}$. To ensure fairness and consistency with the latency experiments presented below, we set $R=8$ and $\mathbb{T}=8 d$. However, due to PISTIS's signature management (see, for example, the optimizations described in Sec. VI-B), PISTIS's worst case delay can be alternatively computed as $\left(3 * 8 * d_{n}\right)+\left(2 * N * d_{p}\right)$. This is in part due to the fact that in PISTIS nodes avoid re-verifying already verified signatures.

Our results, shown in Table I, show that PISTIS has the best worst case latencies of all algorithms for $d_{n}=1 \mathrm{~ms}$ (as mentioned above, in the first column $d_{p}=10$, while in the last two columns $d_{p}$ is such that $1<d_{p}<10$, and can be derived from the numbers provided in the table).

\begin{tabular}{l|c|c|c} 
& {$[9]$} & {$[10]$} & PISTIS \\
\hline$N=25, f=8$ & $2,400 \mathrm{~ms}$ & $26 \mathrm{~ms}$ & $25.6 \mathrm{~ms}$ \\
\hline$N=50, f=16$ & $8,640 \mathrm{~ms}$ & $70 \mathrm{~ms}$ & $27 \mathrm{~ms}$ \\
\hline$N=100, f=33$ & $34,650 \mathrm{~ms}$ & $150 \mathrm{~ms}$ & $30 \mathrm{~ms}$ \\
Table I \\
WORST CASE LATENCIES
\end{tabular}

Two main observations can be made: (1) compared to the other protocols, PISTIS has superior performance due to the fact that PISTIS is event triggered, utilizes fast signature schemes, reduces the number of signatures created and verified, sends fewer messages (which increase individual message failures) and allows processes for fast detection of their tardiness; and (2) PISTIS's expected performance in practice (see Fig. 7) is significantly better than the worst case delay bound reported in the table.

\section{B. Implementation Optimizations}

We implemented three optimizations to improve the performance of PISTIS (as described in Sec. IV-C). (1) If a process $p_{i}$ knows that some process $p_{j}$ has already received $2 f+1$ echo signatures for some message $m, p_{i}$ stops sending echoes related to $m$ to $p_{j}$. Every process implements this optimization by maintaining a list, say $\mathcal{L}$, that contains all the processes from which it has heard $2 f+1$ signatures for a given message. During a broadcast, a process diffuses a message to $X$ processes at random among $\Pi \backslash \mathcal{L}$. Processes do the same for deliver messages. (2) Processes do not verify signatures that they have already received. (3) Processes skip messages that only contain signatures that were already received.

\section{Implementation Configuration and Settings}

We implemented PISTIS in C++ on the Omnet++ 5.4.1 network simulator [11]. In order to accurately measure PISTIS's communication overhead, we configure network links to have a non-limiting $1 \mathrm{Gbps}$ throughput, and a communication latency of either $1 \mathrm{~ms}$ or $5 \mathrm{~ms}$. We evaluated PISTIS's performance using two signature schemes of similar security guarantees, and available in the OpenSSL library [37]: RSA-2048 (i.e., 256 bytes long signatures) and ECDSA with prime256v1 curves (i.e., 71 bytes long signatures). We use broadcast messages of sizes equal to $1 \mathrm{~B}$ and $1 \mathrm{~KB}$.

We run our simulations for systems with $N \in$ $\{25,49,73,300\}$ processes in fully connected networks, and for several values of $X$, which is the number of processes each process forwards a message $m$ to during diffusion. We consider the probability of losing/omitting a message sent at any point in time to be $i / 10$, where $0 \leq i \leq 9$.

\section{PISTIS's Reliability}

To assess PISTIS's reliability, we evaluate the probability that a correct process enters the passive mode. Such probability is a crucial measure: a process becoming passive may lead the system to shutdown and hence to stop delivering messages. Namely, when $N=3 f+1$, a single correct process staying passive for long-enough can, in the worst case (when $f$ Byzantine processes are not sending messages), leave $2 f$ correct processes, which would not be enough to gather quorums of size $2 f+1$, leading those $2 f$ processes to also become passive.

For a given value of $N$ and $p$, we invoke a broadcast at one of the processes and record any non-Byzantine process that crashed itself during broadcast. We obtain our results by repeating each experiment $10^{5}$ times, and we report the probability that a process crashes itself as:

\section{(num. of experiments with self-crashed processes) $/ 10^{5}$}

We study the impact of several parameters, including $\mathbb{T}$, $N, X, f$, and $p$, on PISTIS's reliability, and determine which values should be used to enforce an intended system reliability.

Fig. 3 shows that the system's reliability increases with its size and $\mathbb{T}$ 's value for large enough values of $\mathbb{T} / d$. For example, when $\mathbb{T}=8 d$, a system with 25 (resp. 49) processes operates with high reliability (i.e., there is a negligible probability that a process becomes passive) under message loss rates reaching up to $40 \%$ (resp. $50 \%$ ).

Fig. 4 shows that the actual number of Byzantine processes, which varies between 0 and $f$ (the maximum number of tolerable Byzantine nodes), influences the system's resiliency. As expected, with fewer processes being Byzantine, higher message loss rates are tolerated without any process shutdown.

Impact of the diffusion fanout. In the results presented so far, processes forward each message to $X=f+1$ other random processes. We now study the effect of $X$ by measuring PISTIS's reliability when it varies. Fig. 5 shows that increasing $X$ helps increase the overall system reliability. As expected increasing the fanout (value of $X$ ) reduces the probability of having a non-Byzantine node becoming passive.

Recovery. Fig. 6 details the probability that no Byzantine quorum remains active after a broadcast instance when the message loss probability increases. First, one can observe 


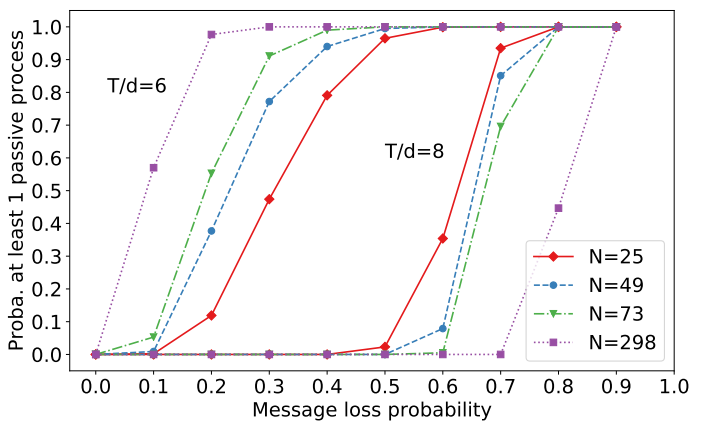

Figure 3. Probability of a correct process becoming passive when $\mathbb{T}=6 d$ or $\mathbb{T}=8 d$, and $X=f+1$ (without recovery)

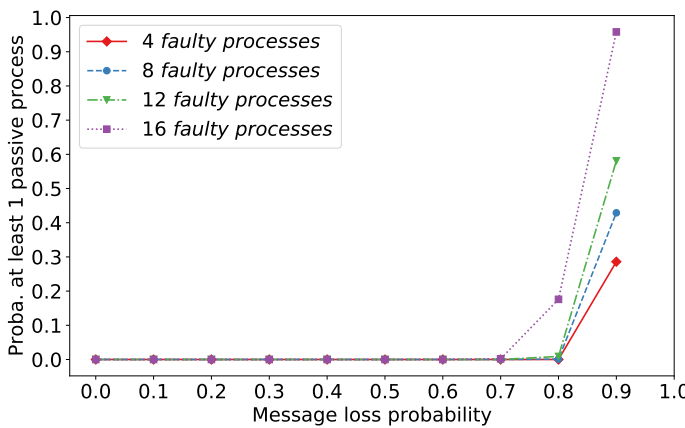

Figure 4. Probability of a correct process becoming passive in a system of 49 processes (i.e., $f=16$ ) using $\mathbb{T}=8 d$ and $X=17$, when $0,4,8,12$ or 16 processes are faulty (without recovery)

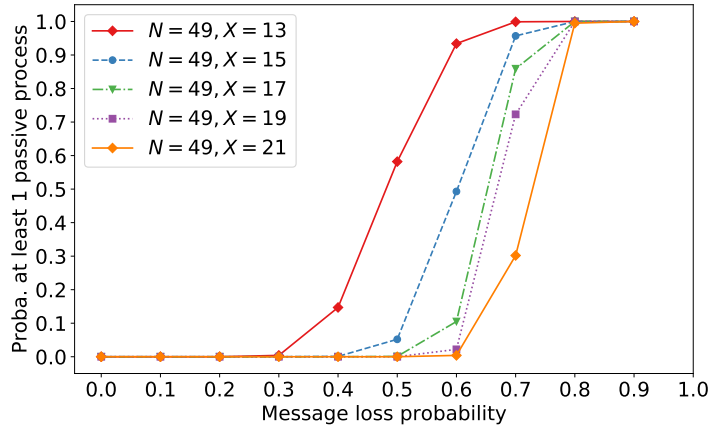

Figure 5. Probability of a correct process becoming passive in a system of 49 processes using $\mathbb{T}=8 d$, and where $X$ varies (without recovery)

that the recovery mechanisms improve the resiliency of the system. For example, with $N=49$, PISTIS can tolerate a $70 \%$ message loss rate without system-wide crashes thanks to the recovery mechanisms, improving over the value of $50 \%$ obtained without recovery. Second, we show that one can further improve the system's tolerance to message losses by overprovisioning the system. By using three more nodes, i.e., 52 in total, the system can tolerate $f=16$ Byzantine nodes and now tolerate up to $80 \%$ of message losses.

\section{E. PISTIS latency and bandwidth consumption}

Next, we evaluate PISTIS's incurred bandwidth and latency. For these experiments, we average results over 1,000 runs. We use $\mathbb{T}=8 d$, since our reliability results show it allows a very large number of message losses to be tolerated. However, we now run our experiments without any message losses to measure the worst case bandwidth consumption. We measure both the protocol latency and bandwidth consumption depending on the value of $X$ that the processes use. We also compare

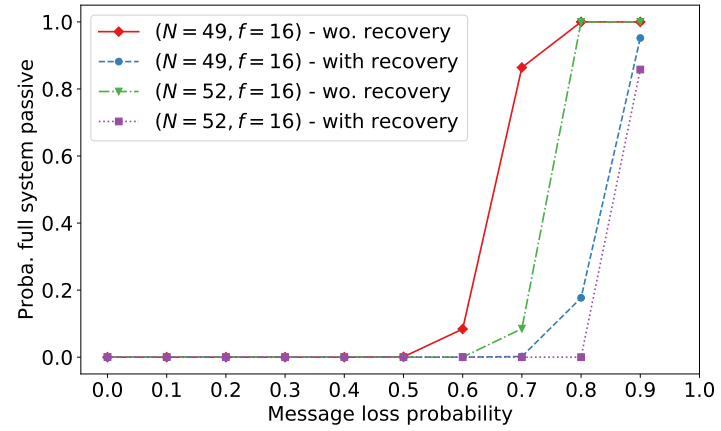

Figure 6. Probability that no Byzantine quorum remains active in systems of 49 or 52 processes, when $\mathbb{T}=8 d, X=17$, and $f=16$ processes are Byzantine.

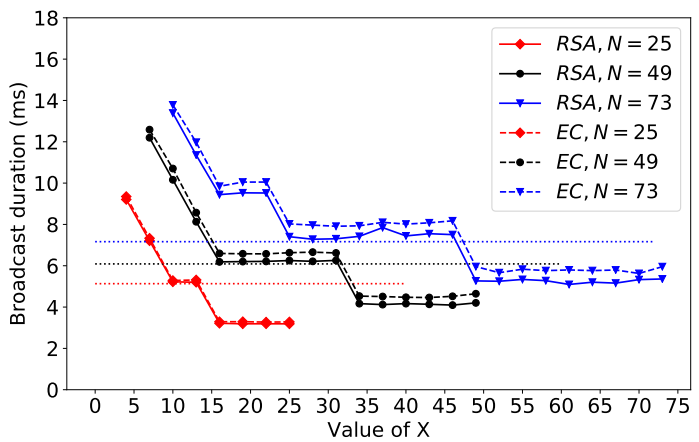

Figure 7. Average latency with a $1 \mathrm{~ms}$ link latency with $\mathbb{T}=8 d$ and without message losses. The dotted lines indicate RT-ByzCast's values [10].

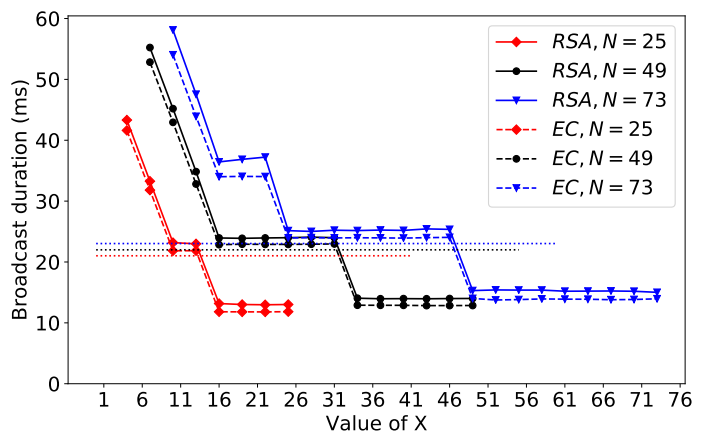

Figure 8. Average latency with a $5 \mathrm{~ms}$ link latency. The dotted lines indicate RT-ByzCast's values [10].

the average latency and bandwidth consumption of PISTIS with that of RT-ByzCast [10]. Note that RT-ByzCast [10] uses ECDSA signatures and all-to-all communication $(X=N)$.

Latency. Fig. 7 and 8 detail the latency for a broadcast message to be delivered by all correct processes in systems of size 25,49 , and 73 (i.e., where $f \in\{8,16,24\}$ ): PISTIS delivers with latencies within $[3 \mathrm{~ms}, 60 \mathrm{~ms}]$ depending on the network delay $d$ and signature scheme used RSA vs. ECDSA. The latency increases when $N$ increases, and decreases when $X$ increases. We draw the following conclusions: (1) PISTIS is slower than RT-ByzCast for $X<f$. For $X \geq f$ PISTIS is on a par with RT-ByzCast until some $X \leq 3 f$ ( $X \leq 2 f$ for systems with up to 400 nodes, see Table II) after which PISTIS is faster; (2) PISTIS's absolute improvement over RTByzcast becomes more significant with increased link delay; (3) When delivering latencies on par with or better than RTByzCast, PISTIS can do so with a lower network overhead as presented next (see Fig. 9 and 10). 


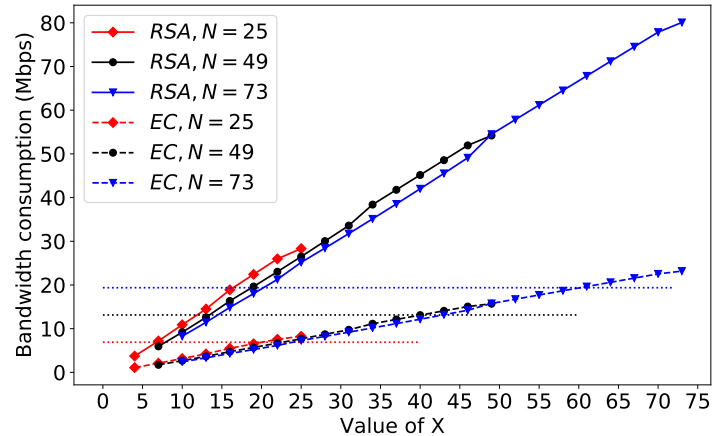

Figure 9. Average bandwidth consumption per node and per communication link with a $1 \mathrm{~ms}$ link latency without message losses. The dotted lines indicate RT-ByzCast's values [10].

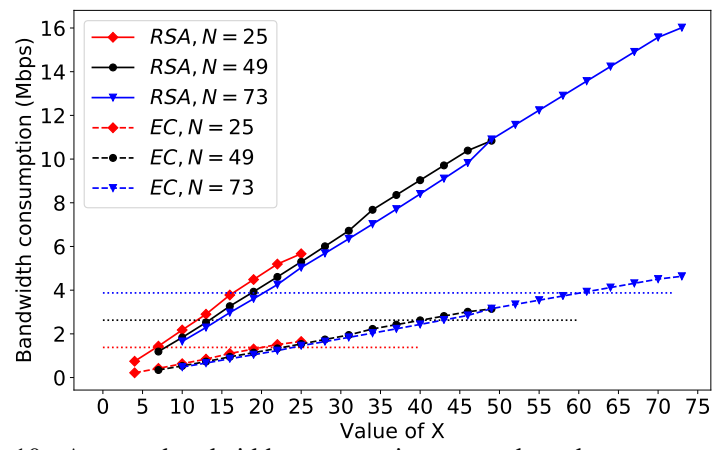

Figure 10. Average bandwidth consumption per node and per communication link with a $5 \mathrm{~ms}$ link latency without message losses. The dotted lines indicate RT-ByzCast's values [10].

Network bandwidth consumption. We now measure PISTIS's bandwidth overhead per broadcast invocation, using RSA and ECDSA signatures. Fig. 9 and 10 present the bandwidth consumption for $1 \mathrm{~B}$ payloads with $1 \mathrm{~ms}$ and $5 \mathrm{~ms}$ link delay, respectively. One can observe that with $X=f+1$ and when using ECDSA signatures, PISTIS's bandwidth consumption is 3.2 times lower than that of RT-ByzCast. We also observe that when using ECDSA signatures there is a fanout between $2 f+1$ and $3 f+1$ such that below this fanout PISTIS's average bandwidth consumption is lower than RT-ByzCast's, while past that threshold, PISTIS's average bandwidth consumption becomes greater than RT-ByzCast's. This is partly due to the fact that PISTIS being event-based sometimes consumes more bandwidth. However, we see in those figures that PISTIS provides a useful trade-off between latency and bandwidth consumption. Fig. 11 shows as well that the bandwidth consumption increases reasonably when the message payload is increased to $1 \mathrm{~KB}$. Besides bandwidth, Fig. 12 (Appx. E) shows that PISITS also sends less message than RT-ByzCast.

Scalability with the system size. We also evaluated how PISTIS' latency and bandwidth consumption evolve with larger system sizes, namely up to 1000 nodes for $X \geq f+1$ and a 5ms link latency. Table II summarizes the results obtained for $X=f+1, X=2 f+1$ and $X=N$. Our results show that PISTIS outperforms RT-ByzCast and provides latencies suitable for (1) fast automatic interactions $(\leq 20 \mathrm{~ms})$ for systems with up to 200 nodes, (2) power systems and substation automation applications $(\leq 100 \mathrm{~ms})$ for systems with up to 1000 nodes, and (3) slow speed auto-

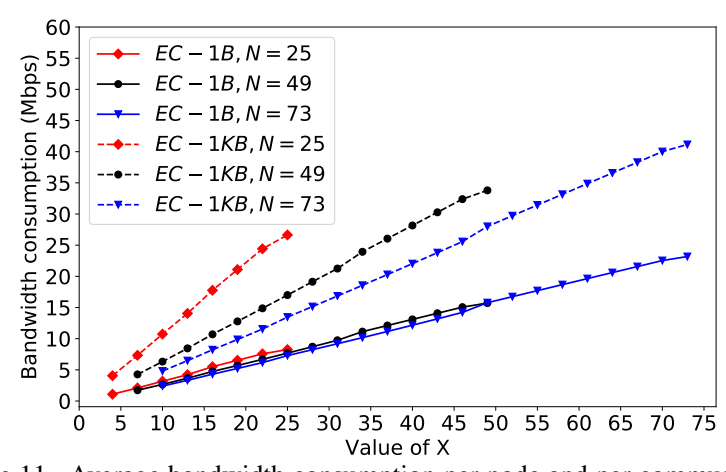

Figure 11. Average bandwidth consumption per node and per communication link with a $1 \mathrm{~ms}$ link latency using either $1 \mathrm{~B}$ or $1 \mathrm{~KB}$ messages, without message losses

\begin{tabular}{l|l|l|l|l||l|l|l|l}
$\mathrm{N}$ & Bdw, & Bdw, & Bdw, & Bdw & Lat, & Lat, & Lat, & Lat \\
& $X_{\min }$ & $X_{\text {mid }}$ & $X_{\max }$ & {$[10]$} & $X_{\min }$ & $X_{\operatorname{mid}}$ & $X_{\max }$ & {$[10]$} \\
\hline 25 & 0.6 & 1.2 & 1.7 & 1.4 & 21.1 & 11.0 & 11.1 & 20.9 \\
49 & 1.0 & 2.2 & 3.1 & 2.6 & 22.3 & 12.4 & 12.0 & 22.0 \\
73 & 1.5 & 3.2 & 4.6 & 3.9 & 23.6 & 13.1 & 13.2 & 23.1 \\
200 & 3.8 & 8.4 & 12.5 & 10.4 & 31.5 & 20.7 & 19.7 & 29.3 \\
300 & 5.7 & 12.5 & 18.6 & 15.6 & 41.2 & 31.2 & 27.4 & 38.0 \\
400 & 7.6 & 16.7 & 25.0 & 20.9 & 59.7 & 43.0 & 32.0 & 41.2 \\
500 & 9.4 & 20.8 & 31.1 & 26.0 & 85.1 & 63.0 & 40.0 & 51.6 \\
1000 & 18.7 & 41.4 & 62.2 & 52 & 296.3 & 213.1 & 98.5 & 116.2 \\
& & \multicolumn{5}{|c}{ Table II }
\end{tabular}

PISTIS BANDWIDTH CONSUMPTION (MBPS) AND BROADCAST DURATION (MS) WITH LARGER SYSTEMS $(f=\lfloor N / 3\rfloor)$, WHERE $X_{\min }=f+1$, $X_{\text {mid }}=2 f+1$ AND $X_{\max }=N$.

control functions $(\leq 500 \mathrm{~ms})$, continuous control applications $(\leq 1 \mathrm{~s})$ and operator commands of SCADA applications $(\leq 2 \mathrm{~s})$ for systems with 1000 nodes or more.

\section{CONCLUSION}

In this paper, we studied how to build large-scale distributed protocols that tolerate network faults and attacks while providing real-time communication. We introduced a suite of proven correct algorithms, starting from a baseline real-time Byzantine reliable broadcast algorithm, called PISTIS, all the way up to real-time Byzantine atomic broadcast and consensus algorithms. PISTIS is empirically shown to be robust, scalable, and capable of meeting timing deadlines of real CPS applications. PISTIS withstands message loss (and delay) rates up to $50 \%$ in systems with 49 nodes and provides bounded delivery latencies in the order of a few milliseconds. PISTIS improves over the state-of-the-art in scalability and latency through its event-triggered nature, gossip-based communications, and fast signature verifications. Our work simplifies the construction of powerful distributed and decentralized monitoring and control applications of various CPS domains, including state-machine replication for fault and intrusion tolerance.

\section{REFERENCES}

[1] J. R. Moyne and D. M. Tilbury. "The Emergence of Industrial Control Networks for Manufacturing Control, Diagnostics, and Safety Data". In: Proc. of the IEEE 95.1 (2007), pp. 29-47.

[2] R. Jacob, M. Zimmerling, P. Huang, J. Beutel, and L. Thiele. "End-to-end Real-time Guarantees in Wireless Cyber-physical Systems". In: RTSS. 2016. 
[3] L. Schenato, B. Sinopoli, M. Franceschetti, K. Poolla, and S. S. Sastry. "Foundations of Control and Estimation Over Lossy Networks". In: Proceedings of the IEEE 95.1 (2007), pp. 163-187.

[4] D. Dzung, R. Guerraoui, D. Kozhaya, and Y.-A. Pignolet. "To Transmit Now Or Not To Transmit Now”. In: SRDS. 2015.

[5] DLC+VIT4IP. D1.1 Scenarios and Requirements Specification. Tech. rep. 2010.

[6] M. M. Patel and A. Aggarwal. "Security attacks in wireless sensor networks: A survey". In: ISSP. 2013.

[7] F. Januário, C. Carvalho, A. Cardoso, and P. Gil. "Security challenges in SCADA systems over Wireless Sensor and Actuator Networks". In: ICUMT. 2016.

[8] P. Polityuk, O. Vukmanovic, and S. Jewkes. Ukraine's power outage was a cyber attack: Ukrenergo. 2017. URL: https:// www . reuters . com/article/us - ukraine - cyber - attack - energy/ ukraines - power - outage - was - a - cyber - attack - ukrenergo idUSKBN1521BA.

[9] F. Cristian, H. Aghili, H. R. Strong, and D. Dolev. "Atomic Broadcast: From Simple Message Diffusion to Byzantine Agreement". In: Inf. Comput. 118.1 (1995), pp. 158-179.

[10] D. Kozhaya, J. Decouchant, and P. Esteves-Verissimo. "RTByzCast: Byzantine-Resilient Real-Time Reliable Broadcast". In: IEEE Trans. Comput. 68.3 (2019), pp. 440-454.

[11] OMNeT++. Last accessed: Feb 24, 2020. URL: https : / / omnetpp.org.

[12] D. Dolev. "Unanimity in an Unknown and Unreliable Environment”. In: FOCS. IEEE Computer Society, 1981, pp. 159-168.

[13] G. Bracha. "Asynchronous Byzantine Agreement Protocols". In: Inf. Comput. 75.2 (1987), pp. 130-143.

[14] P. Verissimo, L. Rodrigues, and M. Baptista. "AMp: A Highly Parallel Atomic Multicast Protocol". In: ACM SIGCOMM. 1989.

[15] R. Guerraoui, P. Kuznetsov, M. Monti, M. Pavlovic, and D.-A. Seredinschi. "Scalable Byzantine Reliable Broadcast". In: DISC, 2019.

[16] A. Babay, J. L. Schultz, T. Tantillo, S. Beckley, E. Jordan, K. Ruddell, K. Jordan, and Y. Amir. "Deploying IntrusionTolerant SCADA for the Power Grid". In: IEEE/IFIP DSN, 2019, pp. 328-335.

[17] A. Babay, T. Tantillo, T. Aron, M. Platania, and Y. Amir. "Network-Attack-Resilient Intrusion-Tolerant SCADA for the Power Grid". In: IEEE/IFIP DSN, 2018.

[18] Y. Amir, B. A. Coan, J. Kirsch, and J. Lane. "Byzantine replication under attack". In: IEEE/IFIP DSN, 2008.

[19] Y. Amir, B. A. Coan, J. Kirsch, and J. Lane. "Prime: Byzantine Replication under Attack". In: IEEE Trans. Dependable Sec. Comput. 8.4 (2011), pp. 564-577.

[20] F. B. Schneider. "Implementing Fault-Tolerant Services Using the State Machine Approach: A Tutorial". In: ACM Comput. Surv. 22.4 (1990), pp. 299-319.

[21] M. Castro and B. Liskov. "Practical Byzantine Fault Tolerance". In: OSDI, 1999.

[22] D. Dzung, R. Guerraoui, D. Kozhaya, and Y.-A. Pignolet. "Never Say Never - Probabilistic and Temporal Failure Detectors". In: IPDPS. 2016.

[23] D. Dolev, C. Dwork, and L. Stockmeyer. "On the Minimal Synchronism Needed for Distributed Consensus". In: JACM 34.1 (1987).

[24] D. Dolev. The Byzantine Generals Strike Again. Tech. rep. Stanford University, CA, USA, 1981.

[25] M. J. Fischer, N. A. Lynch, and M. Merritt. "Easy Impossibility Proofs for Distributed Consensus Problems". In: Distributed Computing 1.1 (1986), pp. 26-39.

[26] S. Viswanathan, R. Tan, and D. K. Y. Yau. "Exploiting Power Grid for Accurate and Secure Clock Synchronization in Industrial IoT”. In: RTSS. 2016.
[27] P. Verissimo and A. Casimiro. "The Timely Computing Base Model and Architecture". In: IEEE Trans. Comput. 51.8 (2002), pp. 916-930.

[28] J. H. An, Y. Dodis, and T. Rabin. "On the Security of Joint Signature and Encryption". In: EUROCRYPT. 2002.

[29] C. Cachin, R. Guerraoui, and L. Rodrigues. Introduction to Reliable and Secure Distributed Programming. SpringerVerlag, 2011.

[30] M. K. Aguilera, C. Delporte-Gallet, H. Fauconnier, and S. Toueg. "On implementing omega in systems with weak reliability and synchrony assumptions". In: Distributed Computing 21.4 (2008), pp. 285-314.

[31] R. Guerraoui, D. Kozhaya, and Y. A. Pignolet. "Right on Time Distributed Shared Memory”. In: RTSS. 2016.

[32] D. Malkhi and M. K. Reiter. "Byzantine Quorum Systems". In: ACM STOC, 1997.

[33] M. Pease, R. Shostak, and L. Lamport. "Reaching Agreement in the Presence of Faults". In: JACM 27.2 (1980), pp. 228-234.

[34] D. Dolev and H. R. Strong. "Authenticated Algorithms for Byzantine Agreement". In: SIAM J. Comput. 12.4 (1983), pp. 656-666.

[35] D. Dolev and R. Reischuk. "Bounds on Information Exchange for Byzantine Agreement". In: JACM 32.1 (1985), pp. 191204.

[36] L. Lamport, R. Shostak, and M. Pease. "The Byzantine Generals Problem". In: ACM Transactions on Programming Languages and Systems 4/3 (1982), pp. 382-401.

[37] OpenSSL. Last accessed: Feb 25, 2020. URL: https://www. openssl.org/.

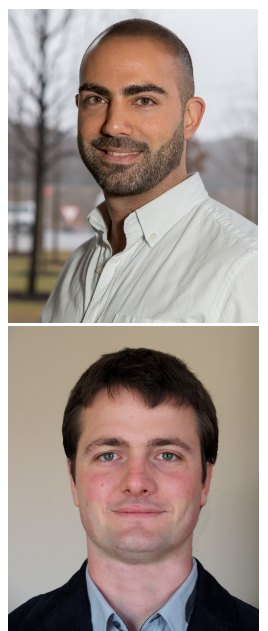

David Kozhaya is a Senior Scientist at ABB Research, Switzerland. He received his $\mathrm{PhD}$ degree in Computer Science in 2016, from EPFL, Switzerland, where he was granted a fellowship from the doctoral school. His primary research interests include reliable distributed computing, real-time distributed systems, and fault- and intrusion-tolerant distributed algorithms.

Jérémie Decouchant is an Assistant Professor at TU Delft, the Netherlands. He received his Ph.D in Computer Science in 2015 from the Grenoble-Alpes University, France. His research interests include resilient distributed computing, privacy-preserving systems, and their application to Blockchain, genomics, and machine learning.

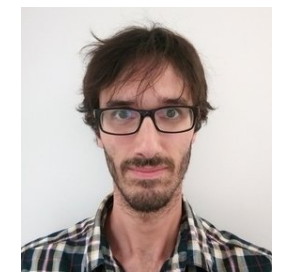

Vincent Rahli is a Senior Lecturer at the University of Birmingham. He received his Ph.D in Computer Science from Heriot-Watt University, UK. His research focuses on designing, formalizing, and using type theories and on the verification of distributed systems using proof assistants.

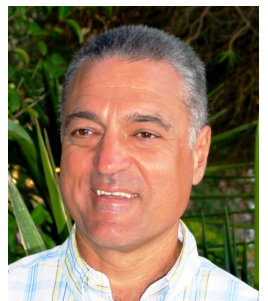

Paulo Esteves-Veríssimo is a professor at the KAUST University (KSA), and Director of the Resilient Computing and Cybersecurity Center (RC3 https://rc3.kaust.edu.sa/). He was a member of the Sci\&Tech. Comm. of ECSO EU Cyber Security Organisation, Chair of IFIP WG 10.4 on Dependable Comp. and F/T, and vice-Chair of the Steer. Comm. of the DSN conference. He is Fellow of IEEE and of $\mathrm{ACM}$, and associate editor of the IEEE TETC journal, author of over 200 peer-refereed publications and co-author of 5 books. He is currently interested in resilient computing, and its potential to improve classic cybersecurity techniques, in: SDN-based infrastructures; autonomous vehicles from earth to space; distributed control systems; digital health and genomics; or blockchain and cryptocurrencies. 


\section{APPENDIX A \\ DIFFERENCES BETWEEN PROBABILISTIC SYNCHRONY AND OTHER STANDARD MODELS}

a) Comparison with fully asynchronous models: Our model is more informative than traditional fully asynchronous models. More precisely, asynchronous models do not make any assumptions regarding message transmission and processing delays, while we assume that messages are delivered within a maximum transmission delay $d$ with high probability.

b) Comparison with synchronous models: Our communication model is a probabilistic synchronous one. We recall that in every transmission attempt a link may (with some probability) violate reliability and timeliness by dropping the message or delivering it within a delay $>d$. In case of message loss (omission) a sender that needs to re-transmit that message again faces yet another risk of transmission failure. Due to omissions (losses in consecutive transmission attempts) and the required follow-up re-transmissions, the time it takes to send a message reliably from one process to another (measured from the time of the first transmission attempt) may be unbounded. So, despite links being reliable and timely with high probability, our communication system is no longer synchronous.

c) Comparison with partially synchronous models: In comparison with partial synchrony [1], which assumes that communication becomes forever synchronous after some unknown point in time, our probabilistic synchronous model guarantees only finite synchronous periods (with variable durations) that may occur randomly during the lifetime of the system. In fact such probabilistic synchronous communication has been shown to be weaker, in some sense [2], than partial synchrony. For example, while the celebrated failure detectors of [3] can be implemented in partially synchronous systems they are impossible to implement in the systems with probabilistic synchronous communication [2].

d) The need for probabilistic synchrony models: Probabilistic synchronous models (such as the one presented here or $[2,4])$ are more "realistic" than synchronous models in the sense that timing assumptions cannot always be ensured in distributed systems because, for example, of the difficulty of guaranteeing reliable communication between the nodes of a system. Making the probability of timing failures (e.g., that messages might be delivered after $d$ ) transparent to the model and protocols makes them more robust. For example, it allows designing protocols where messages might not always arrive within a specified maximum transmission delay. Systems that require processes to operate in a timely fashion, such as mission critical systems, can therefore dynamically adapt to such untimely situations to ensure that timing guarantees are fulfilled.

e) Comparison with quasi-synchronous models: Quasisynchronous models [4] address the timing issues mentioned above. In [4] synchronism is characterized by the following properties: $\mathrm{P} 1$ - processing speeds are bounded and known; P2 - message delivery delays are bounded and known; P3local clock rate drifts are bounded and known; P4-load patterns are bounded and known; and P5-differences among local clocks are bounded and known. A system is quasi- synchronous if it satisfies properties $\mathrm{P} 1-\mathrm{P} 5$, and at least one of those does not hold with some known non-zero probability. As in a quasi-synchronous model, in our probabilistic model P2 only holds with high probability. Note, however, that in our probabilistic model we do not assume that differences among local clocks are bounded and known.

\section{APPENDIX B}

\section{CorrectNess of PISTIS (AlgORITHM 2)—ProOF OF} THEOREM 1

Lemma 1 (Validity). If a correct process $p_{i}$ broadcasts $m$ then $p_{i}$ eventually delivers $m$.

Proof outline. Because $p_{i}$ is correct, it will hear echoes of $m$ from $2 f+1$ processes (including $p_{i}$ ) by $t+\mathbb{T}$, where $t$ is the time $p_{i}$ broadcasted $m$. This is true as otherwise, i.e., if less than $2 f+1$ echoes for $m$ are heard, $p_{i}$ would kill itself (hence is no longer correct). Indeed, $p_{i}$ triggered a timer (see line 49 of Algorithm 2) when it started broadcasting $m$ (see line 6). Because $p_{i}$ received $2 f+1$ echoes for $m$, it must have delivered $m$ too (see lines 14, 19, and 26 of Algorithm 2).

Lemma 2 (No duplication). No correct process delivers message $m$ more than once.

Proof outline. According to line 60 of Algorithm 2 a process only delivers a message if the corresponding $\mathcal{R}_{\text {deliver }}$ does not exist, and creates one right after delivering, thereby preventing from delivering a message twice.

Lemma 3 (Integrity). If some correct process $p_{j}$ delivers a message $m$ with correct sender $p_{i}$, then $m$ was previously broadcasted by $p_{i}$.

Proof outline. Because $p_{j}$ delivered $m$, it must have received $2 f+1$ signed echoes for $m$ (see lines 14, 19, 26, and 33 of Algorithm 2). As mentioned in Remark 1, an echo message is not handled unless it is signed by the claimed sender. More precisely, upon receipt of a message of the form Echo $\left(\left\langle p_{i}, s q, v\right\rangle, \Sigma\right)$ or Deliver $\left(\left\langle p_{i}, s q, v, \Sigma\right\rangle, \Sigma^{\prime}\right), p_{j}$ only handle the message if $\Sigma$ contains a signature from $p_{i}$. Now, because the sender $p_{i}$ is correct, it must have indeed sent an echo message for $\left\langle p_{i}, v\right\rangle$. Finally, we prove by induction on the chain of local events happening at $p_{i}$ (a correct process) that led to this message being sent, that $p_{i}$ must have broadcasted it.

Lemma 4 (Intersecting delivery). Let $p$ be a correct process that starts delivering some message $m$ at some time $t_{d}$. Then, there exists a collection $B$ of $2 f+1$ processes such that all correct processes in $B$ only deliver $m$ for a full $\mathbb{T}$ duration starting some time prior to $t_{d}+\mathbb{T}$.

Proof outline. Let us first point out that because $p$ starts delivering at $t_{d}$, and because it is correct, $2 f+1$ processes must have received this deliver message by $t_{d}+\mathbb{T}$ (otherwise $p$ would kill itself because it wouldn't be connected-the proofof-connectivity is executed in piggyback mode). Let $A$ be this collection of $2 f+1$ processes (note that $p \in A$ ). For each correct process $q \in A, q$ must have started delivering some time prior to $t_{d}+\mathbb{T}$.

Let us now prove this lemma by induction on $t_{d}$.

Either a correct process within $A$ started delivering prior to $t_{d}$ or not. If one did, in which case $t_{d}>0$, then we conclude by our induction hypothesis. Otherwise all correct nodes in $A$ (at least $f+1$ ) are only delivering starting from $t_{d}$. Because they start delivering prior to $t_{d}+\mathbb{T}$, and because they deliver for $2 \mathbb{T}$, it must be that all correct processes within that collection 
only deliver $m$ for a full $\mathbb{T}$ duration starting at most by $t_{d}+\mathbb{T}$ (until at most $t_{d}+2 \mathbb{T}$ ).

Lemma 5 (Timely agreement). If a correct process $p_{i}$ broadcasts $m$ at real time $t$, then all correct processes deliver $m$ by $t+3 \mathbb{T}$.

Proof outline. Since $p_{i}$ is correct during this broadcast, then it must have received $2 f+1$ echoes for $m$ and must then have started delivering $m$ at $t_{d} \in[t, t+\mathbb{T}]$. By Lemma 4, there exists a collection $B$ of $2 f+1$ processes such that all correct processes in $B$ only deliver $m$ for a full $\mathbb{T}$ duration starting some time prior to $t_{d}+\mathbb{T}$. Now, every other correct process $p_{j}$ must be connected to $2 f+1$ processes in any proofof-connectivity period $p c=\left[t_{0}, t_{0}+\mathbb{T}\right]$-let $C(p c)$ denote those $2 f+1$ processes. Therefore, because there are $3 f+1$ processes, there must be a correct process, say $r$, and a proofof-connectivity period $p c=\left[t_{j}, t_{j}+\mathbb{T}\right]$ at $p_{j}$ such that: (1) $r$ is in the intersection of $B$ and $C(p c)$ (there must be at least one correct process in that intersection because it is of size $f+1$ ); and such that (2) $p_{j}$ received $m$ during $p c$ from $r$, which sent it at most by $t_{d}+2 \mathbb{T}$. Therefore, $p_{j}$ must have delivered by $t+3 \mathbb{T}$.

Lemma 6 (Agreement). If some correct process $p_{i}$ delivers $m$, then all correct processes eventually deliver $m$.

Proof outline. This is a straightforward consequence of Lemma 5.

Lemma 7 (Timeliness). If a correct process $p_{i}$ broadcasts $m$ at real time $t$, then no correct process delivers $m$ after $t+3 \mathbb{T}$.

Proof outline. This is a straightforward consequence of Lemma 5.

\section{APPENDIX C \\ Correctness of PISTIC-CS-PROOF OF THEOREM 2}

Recall that since Algorithm $\mathcal{A}$ implements interactive consistency, then when $\mathcal{A}$ eventually terminates all correct processes will have the same vector of proposals where the values relative to correct processes are indeed what these correct processes have proposed. In fact interactive consistency [5] guarantees the two following properties:

IC.1 The non-faulty processors compute exactly the same vector.

IC.2 The element of this vector corresponding to a given nonfaulty processor is the private value of that processor.

We now prove Thm. 2, i.e., that assuming algorithm $\mathcal{A}$ implements interactive consistency in a known bounded number of communication rounds (this is used to prove Lemma 11), as well as Assumptions 1 and 2, then $\mathcal{A}$ implements RTBC (see Sec. V-A) in our system model (see Sec. III).

Lemma 8 (RTBC-Termination). Every correct process eventually decides.

Proof outline. By Assumption 1, a correct process $p_{i}$ accesses the network only through the RTBRB primitive. Therefore, because $p_{i}$ is correct and therefore does not enter passive mode while executing RTBRB, it must terminate. By IC. $1 p_{i}$ must compute a vector. Finally, $p_{i}$ will apply the deterministic function described in Assumption 2 to that vector to obtain a value $v$, which is the value $p_{i}$ decides upon.
Lemma 9 (RTBC-Agreement). No two correct processes decide differently.

Proof outline. Let $p_{i}$ be a correct process that decides upon a value $v_{i}$, and $p_{j}$ be a correct process that decides upon a value $v_{j}$. Again, by Assumption $1, p_{i}$ and $p_{j}$ must not enter passive mode while using the RTBRB primitive. By IC.1, $p_{i}$ and $p_{j}$ must compute the same vector $V$. Both $p_{i}$ and $p_{j}$ apply the deterministic function described in Assumption 2 to this vector $V$. Therefore, $v_{i}$ must be equal to $v_{j}$.

Lemma 10 (RTBC-Validity). If all correct processes propose the same value $v$, then any correct process that decides, decides $v$. Otherwise, a correct process may only decide a value that was proposed by some correct process or the special value $\perp$.

Proof outline. First, note that by Assumption 1, correct processes must not enter passive mode while using the RTBRB primitive. Now, if all correct processes propose the same value $v$, then by IC. 2 the obtained interactive consistency vector computed by a correct process should contain $v$ a number of times equal to the number of correct processes, i.e., at least $2 f+1$ times. Finally, since all correct processes apply the deterministic function described in Assumption 2 to their vectors, they must all decide on $v$.

Let us now assume that not all correct processes propose the same value $v$. If a correct process $p$ decides upon a value $v^{\prime}$ then by Assumption 2, it must be that either (1) its interactive consistency vector contains at least $2 f+1$ times this value $v^{\prime}$; or (2) that $v^{\prime}$ is the special value $\perp$. In case $v^{\prime}$ appears $2 f+1$ times in $p$ 's interactive consistency vector, then by IC. 2 , it must be that $v^{\prime}$ was proposed by a correct process. This concludes the proof.

Lemma 11 (RTBC-Timeliness). If a correct process $p_{i}$ proposes a value to consensus at time $t$, then no correct process decides after $t+\Delta_{\mathrm{C}}$.

Proof outline. The way we implement consensus is first by reaching interactive consistency and applying a deterministic function after. The deterministic function is a computational load that requires scanning the consistency vector and hence has a known bounded duration since we assume that correct processes are synchronous. Therefore, it is sufficient to prove that the interactive consistency protocol finishes in a bounded duration (in the sense that correct processes compute their interactive consistency vectors in a bounded amount of time).

Recall that we assume that Algorithm $\mathcal{A}$ requires a bounded number of communication rounds to terminate, say $k$. By Assumption 1 processes send and receive messages over the network only via the RTBRB primitive. Hence any communication round has a bounded duration, that being a multiple, say $m$, of $\Delta_{R}$, the duration needed by the RTBRB primitive to complete (which is at most $3 \mathbb{T}$ ). Therefore, because by Assumption 1, correct processes must not enter passive mode while using the RTBRB primitive, it must be that correct processes will decide before $t+(k \times m \times 3 \mathbb{T})$, which concludes our proof.

\section{APPENDIX D}

CORRECTNESS OF PISTIC-AT-PROOF OF THEOREM 3

\section{A. Reduction to RTBAB}

To prove Thm. 3 , we only have to prove that Algorithm $\mathcal{A}$ satisfies the RTBAB-Timeliness property.

Proof outline. Let us assume that the correct process $p_{i}$ RTBAB-broadcasts $m$ at time $t$. We have to prove that no correct process RTBAB-delivers $m$ after real time $t+\Delta_{\mathrm{A}}$, 
for some $\Delta_{A}$. We prove this by proving the stronger result that there exists a $\Delta_{\mathrm{A}}$ such that all correct processes RTBABdeliver $m$ by $t+\Delta_{\mathrm{A}}$.

By Property $1, p_{i}$ RTBRB-broadcasts $m$ with some sequence number $s e q_{t}$ by time $t+\Delta_{\mathrm{B}}$. By RTBRB-Validity, RTBRB-Timeliness and RTBRB-Agreement, all correct processes RTBRB-deliver $m$ by some time $t+\Delta_{\mathrm{B}}+\Delta_{\mathrm{R}}$. By Property 2, all correct processes will RTBC-propose or RTBCdecide $m$ by $t+\Delta_{\mathrm{B}}+\Delta_{\mathrm{R}}+\Delta_{\mathrm{P}}$.

If one correct process RTBC-decides $m$ by $t+\Delta_{B}+\Delta_{R}+\Delta_{P}$, then by the RTBC properties, all correct processes will RTBCdecide by $t+\Delta_{B}+\Delta_{R}+\Delta_{P}+\Delta_{C}$, and by Property 4 , they will RTBAB-deliver by $t+\Delta_{B}+\Delta_{R}+\Delta_{P}+\Delta_{C}+\Delta_{D}$, which concludes our proof. Therefore, let us now consider the case where they all RTBC-propose $m$ by $t+\Delta_{\mathrm{B}}+\Delta_{\mathrm{R}}+\Delta_{\mathrm{P}}$.

However, it might be that they RTBC-propose $m$ in different RTBC instances. We want to prove that there will be an RTBC instance inst $_{m}$ where "enough" correct nodes RTBC-propose $m$ at that instance, by time $t+\Delta_{m}$ (for some fixed $\Delta_{m}$ ), so that it results in inst $_{m}$ deciding $m$. Then, by RTBC-Termination, RTBC-Agreement, RTBC-Timeliness, and Property 4, we can conclude that all correct processes RTBAB-deliver $m$ by time $t+\Delta_{m}+\Delta_{\mathrm{C}}+\Delta_{\mathrm{D}}$. Let us now prove that such an instance inst $_{m}$ indeed exists.

Because all correct processes RTBC-propose $m$ by $t+\Delta_{\mathrm{B}}+$ $\Delta_{R}+\Delta_{P}$, there must be a greatest instance inst $g$ such that a correct process $p_{g}$ RTBC-proposes $m$ at some time $t_{k} \leq$ $t+\Delta_{\mathrm{B}}+\Delta_{\mathrm{R}}+\Delta_{\mathrm{P}}$. Now, either (1) $m$ was RTBC-decided at a prior instance inst $_{p}$ (by all correct processes, by the RTBC properties), or (2) not. In case it was (i.e., case (1)), all correct processes must have RTBC-decided $m$ by time $t+\Delta_{\mathrm{B}}+\Delta_{\mathrm{R}}+$ $\Delta_{\mathrm{P}}+\Delta_{\mathrm{C}}$ by the RTBC properties and because inst $_{p}$ must have been dealt with by $p_{g}$ before inst $t_{g}$ by Property 6 . Now, by Property 4 , it must be that all correct processes must have RTBAB-delivered $m$ by time $t+\Delta_{\mathrm{B}}+\Delta_{\mathrm{R}}+\Delta_{\mathrm{P}}+\Delta_{\mathrm{C}}$.

Let us now focus on case (2), i.e., $m$ was not RTBC-decided at a prior instance. By Property 3, correct processes must be RTBC-proposing either $m$ or $\perp$ at instance inst $_{g}$. Let us prove that they cannot propose $\perp$, in which case we conclude using RTBC-Validity and Property 4 , and $\Delta_{\mathrm{A}}$ is again $t+\Delta_{\mathrm{B}}+\Delta_{\mathrm{R}}+$ $\Delta_{\mathrm{P}}+\Delta_{\mathrm{C}}$. We prove that correct processes cannot propose $\perp$ at instance inst $_{g}$ by contradiction. Let us assume that some correct process $p_{j}$ votes for $\perp$ at instance inst $_{g}$ (therefore, $p_{j}$ cannot be $p_{g}$ ). By definition of inst $t_{g}$, it must be that $p_{j}$ votes for $m$ at a prior instance inst $_{p}$. Because it is an instance prior to inst $_{p}$, as mentioned above, $m$ was not RTBC-decided at that instance. Therefore, by Property 3, and RTBC-Validity, it must be that this instance ended up in $\perp$ being decided. Finally, we obtain a contradiction from the fact that $p_{j}$ must also RTBC-propose $m$ at instance inst $_{g}$, which we prove by induction on the list of instances between inst $_{p}$ and inst $_{g}$ and using Property 5.

\section{B. PISTIS-AT: a Class of Algorithms Implementing RTBAB}

Algorithm 3 provides an example of a PISTIS-AT algorithm, which implements the RTBAB primitive presented in Sec. V-B. We assume here that a process broadcasts a message by invoking RTBAB-broadcast(), and delivers a message invoking $\mathrm{RTBAB}-\operatorname{deliver().~In~addition,~}$ RTBAB-init(rtbab) instantiates a new instance of RTBAB with id rtbab. To guarantee total order, each process maintains a monotonically increasing sequence number $s e q$, which is incremented every time RTBAB-broadcast () is called.
Lemma 12. Given an RTBAB instance inst, such that $p_{i}$ is the leader of inst, all correct processes will either RTBC-propose a value received from $p_{i}$ or $\perp$ (in case they have not received any new message from $p_{i}$ since the last one they processed). Moreover, given two correct processes that RTBC-propose such values at instance inst, it must be that either those values are equal (to the $k^{\text {th }}$ new value broadcasted by $p_{i}$, for some $k$ ) or one of them is $\perp$ (in case the corresponding process has not received $p_{i}$ 's $k^{\text {th }}$ broadcasted new value yet, and has already processed all previous broadcasted value from $p_{i}$ ).

Proof outline. This can be proved by induction on causal time.

The first time those correct processes RTBC-propose a value at an instance such that $p_{i}$ is the leader, it must be that either this value is the first value RTBAB-broadcasted by $p_{i}$, or $\perp$.

The inductive case goes as follows: we assume that our property is true at a given instance inst such that $p_{i}$ is the leader, and where correct processes RTBC-propose either $v$ (the $(k-1)^{t h}$ new value proposed by $\left.p_{i}\right)$ or $\perp$, and we prove that the property is still true at the next such instance inst ${ }^{\prime}$. By RTBC-Validity, it must be that correct processes either RTBCdecide $v$ or $\perp$, and by RTBC-Agreement, they must not decide differently. Therefore, if they decide $v$ at instance inst, then $v$ will be added to the delivered set, and therefore never added to unordered again; and in addition, it will be removed from unordered. At the next instance inst', these processes will vote either for the $k^{t h}$ new value proposed by $p_{i}$ or for $\perp$ if they have not received that $k^{t h}$ new value. In particular, if one of those correct processes RTBC-proposed $\perp$ because it had not received $v$ yet, then at instance inst $^{\prime}$ it will either propose the $k^{\text {th }}$ new value proposed by $p_{i}$ (since $v$ is skipped because already delivered), or $\perp$ in case it has not received this $k^{\text {th }}$ new value yet. Otherwise if they decide $\perp$, then the correct processes that voted for $v$ will still vote for $v$ at inst', and those that voted for $\perp$ will either keep on voting for $\perp$ if they still have not received $v$, or finally receive $v$ and start voting for $v$. Note that by RTBAB-Agreement, all correct processes must eventually receive $v$.

In order to obtain time bounds that do not depend on Algorithm 3's variable, we make the following assumption:

Assumption 3. Correct processes wait for $\Delta_{R}+\Delta_{W}+(n \times$ $\left.\left(\Delta_{\mathrm{C}}+\Delta_{\mathrm{W}}\right)\right)$ between two different broadcasts.

As we will see below, this is the time it takes to guarantee that all correct processes RTBAB-deliver an RTBRBbroadcasted value.

\section{Lemma 13. Algorithm 3 satisfies Property 1.}

Proof outline. Property 1 holds because Algorithm 3 RTBTBbroadcasts messages on each call to RTBAB-broadcast (see lines 5 and 6 of Algorithm 3).

Lemma 14. Algorithm 3 satisfies Property 4.

Proof outline. If a value $v$ (different from $\perp$ ) is RTBCdecided at time $t$, and the RTBC instance is the current instance, and $v$ is not in delivered, then it is RTBAB-delivered. If $v$ is in delivered, then it must be that it was added to that set in the past, in which case it was delivered at that time.

Now, if the RTBC instance is not the current instance, Algorithm 3 retries handling the messages after a while. The number of times a process will retry handling deliver messages is bounded because instances are handled in a monotonic order and are bounded in time according to RTBC-Timeliness.

Lemma 15. Under Assumption 3, Algorithm 3 satisfies Property 2. 
Proof outline. First of all, let us point out that RTBRB-deliver messages are treated in monotonic order. Let us now consider three cases. In the following, we first provide variabledependent bounds, and we then explain how to get independent bounds using Assumption 3.

Case (1): Whenever a process $p_{i}$ receives a RTBRB-deliver message $m$ at time $t$ with sequence number num, broadcasted by $p_{j}$, which is the next one to receive (i.e., $n u m=n \operatorname{ext}\left[p_{j}\right]$ ), and if $m$ is not already in delivered, then $p_{i}$ will append $m$ to its unordered $\left[p_{j}\right]$ list. We now have to prove that $m$ will then be RTBC-proposed or RTBC-decided by some time $t+\Delta_{\mathrm{P}}$, for some bounded $\Delta_{\mathrm{P}}$. Because $m$ is now in $p_{i}$ 's unordered $\left[p_{j}\right]$ list, the event line 18 will be triggered at least until $m$ is removed from the list. Because Algorithm 3 uses the rotating coordinator paradigm, then a value broadcasted by some process $p_{k}$ is voted upon using an RTBC instance only every $n$ (the total number of processes) instances (i.e., whenever $p_{k}$ is the leader). However, there might be other values before $m$ in the unordered $\left[p_{j}\right]$ lists maintained by the processes. The processes have to RTBC-decide these previous values to start RTBC-proposing $m$ if $m$ has not been RTBAB-delivered in the meantime (otherwise we can conclude because RTBAB-delivered messages are RTBC-decided upon). Because of the rotating coordinator scheme, and by the RTBC properties and Lemma 12, we get the guarantee that $m$ will be RTBC-proposed by $t+\left(n \times\left(\Delta_{\mathrm{C}}+\Delta_{\mathrm{W}}\right) \times(n u m+1)\right)$, where $\Delta_{\mathrm{C}}+\Delta_{\mathrm{W}}$ is the time it takes to complete an RTBC instance, and $n \times\left(\Delta_{C}+\Delta_{\mathrm{W}}\right)$ is the time it takes to rotate through the leaders $\left(\Delta_{\mathrm{W}}\right.$ is the time processes wait for before re-trying to handle a message-see line 15 and line 36). Now, thanks to Assumption 3, we can derive that all previous values stored in unordered $\left[p_{j}\right]$ have already been decided upon when correct processes deliver $m$. Therefore, we get that $m$ will be RTBCproposed by $t+\left(n \times\left(\Delta_{\mathrm{C}}+\Delta_{\mathrm{W}}\right)\right)$.

Case (2): If $m$ is already in delivered, then $p_{i}$ must have already RTBC-decided $m$ according to lines 28-31.

Case (3): If $m$ is not the next value that $p_{i}$ is supposed to receive, it will re-try RTBRB-delivering $m$ after $\Delta_{\mathrm{W}}$ until it has received all the previous values. The RTBRB properties guarantee that if some correct process $p_{j}$ broadcasts a value $v$ at time $t$, then correct processes will deliver $v$ by $t+\Delta_{\mathrm{R}}+\Delta_{\mathrm{W}}$. Therefore, it must be that correct processes will have stored $m$ (and all previous values) in their unordered $\left[p_{j}\right]$ list by $t+\left(\Delta_{\mathrm{R}}+\Delta_{\mathrm{W}}\right) \times($ num +1$)$. Finally, following the same argument as above, we get that $m$ will be RTBC-proposed by $t+\left(\left(\Delta_{\mathrm{R}}+\Delta_{\mathrm{W}}\right) \times(n u m+1)\right)+\left(n \times\left(\Delta_{\mathrm{C}}+\Delta_{\mathrm{W}}\right) \times(n u m+1)\right)$. As mentioned above, thanks to Assumption 3, we can derive that all previous values stored in unordered $\left[p_{j}\right]$ have already been RTBRB-delivered and RTBC-decided upon when correct processes deliver $m$. Therefore, we get that $m$ will be RTBCproposed by $t+\left(\left(\Delta_{R}+\Delta_{W}\right)\right)+\left(n \times\left(\Delta_{C}+\Delta_{W}\right)\right)$.

Lemma 16. Algorithm 3 satisfies Property 3.

Proof outline. This is a straightforward consequence of Lemma 12.

\section{Lemma 17. Algorithm 3 satisfies Property 5.}

Proof outline. Let $p_{i}$ be a correct process that proposes a value $v$, with broadcaster $p_{j}$, at a given time $t$, using a given RTBC instance inst, and such that this instance does not decide $v$. By Lemma 12, all correct processes propose $v$ or $\perp$ at that instance. By the RTBC properties, because inst does not decide $v$, it must decide $\perp$. Therefore, $p_{i}$ will increment its RTBC instance number but will keep $m$ at the head of its unordered $\left[p_{j}\right]$ list. After a full rotation through the leaders, it will RTBC-propose $v$ again at the later instance inst $+n$, where $0<n$.
Moreover, no correct process will propose $v$ between inst and inst $+n$ because $p_{j}$ ( $v$ 's broadcaster) is the leader of inst and inst $+n$ but not of the instances in between, and $v$ can only be in the unordered $\left[p_{j}\right]$ lists.

Lemma 18. Algorithm 3 satisfies Property 6.

Proof outline. By design, correct processes RTBC-propose exactly one value per RTBC instance because they only start proposing a value in a new instance if busy is False; in which case they set busy to True; wait for this instance to complete; and finally increment the RTBC instance number and set back busy to False.

Correct processes propose values in all RTBC instances and monotonically because they increment the the RTBC instance number by one every time an RTBC instance complete.

Finally, correct processes do not run RTBC instances in parallel thanks to the busy flag.

\section{Direct Proof of Algorithm 3's Correctness}

Lemma 19 (RTBAB-Validity). If a correct $p_{i}$ process broadcasts $m$, then $p_{i}$ eventually delivers $m$.

Proof outline. By RTBRB-Validity, $p_{i}$ eventually delivers $m$ with sequence number num. If $p_{i}$ has already delivered $m$, i.e., $m \in$ delivered, then we are done. Otherwise, because $p_{i}$ broadcasts messages monotonically (and without gaps), it will append $m$ to its list of unordered messages (line 13 of Algorithm 3). Therefore, line 18 will be triggered until $m$ is removed from the list, as long as $p_{i}$ eventually resets busy to False once it has set it to True, which is true by RTBC-termination. When finally $p_{i}$ is the leader of its current instance, say inst $t_{1}$, and that $m$ is at the head of $p_{i}$ 's unordered list, $p_{i}$ will RTBC-propose $m$. By RTBC-Validity, either all the correct processes RTBC-propose $m$, in which case $p_{i}$ delivers $m$; or some correct processes RTBC-propose values different from $m$. As mentioned above, such proposed values must then be $\perp$, in which case $p_{i}$ might RTBC-decide $m$ or $\perp$. Again as mentioned above, if $p_{i}$ does not deliver $m$, it will again either decide $m$ or $\perp$ at the next instance where it is the leader. Because by RTBAB-Agreement, all correct processes eventually receive $m$, it must be that eventually, $p_{i}$ RTBCdecides $m$ for an instance where it is the leader, and in turn RTBAB-deliver $m$.

Lemma 20 (RTBAB-No duplication). No message is delivered more than once.

Proof outline. This property straightforwardly follows trivially from the fact that delivered values are added to the delivered set line 31 , and from the fact that a process always checks whether it has delivered a message $m$ before delivering $m$ (see line 30).

Lemma 21 (RTBAB-Integrity). If some correct process delivers a message $m$ with initial sender $p_{i}$ and process $p_{i}$ is correct, then $m$ was previously broadcast by $p_{i}$.

Proof outline. First of all, the RTBAB-delivered value $m$ (which must be different from $\perp$ ) with sender $p_{i}$ (i.e., such that $p_{i}$ is the leader of the current instance) must have been RTBCdecided upon. By RTBC-Agreement and RTBC-Termination, it must be that the correct sender $p_{i}$ has also RTBC-decided upon $m$. It must be that $m$ was it $p_{i}$ 's own unordered list. Therefore, it must be that $p_{i}$ RTBRB-delivered $m$. Finally, by RTBRB-Integrity, it must be that $p_{i}$ previously broadcasted $m$.

Lemma 22 (RTBAB-Agreement). If some message $m$ is delivered by any correct process, then every correct process eventually delivers $m$. 
Proof outline. Let $p_{i}$ be the process that RTBAB-delivered $m$ at instance inst, such that $p_{l}$ is the leader of that instance. This delivered value must be different from $\perp$, and must have been RTBC-decided upon. By RTBC-Agreement and RTBCTermination, it must be that all correct processes eventually RTBC-decide $m$ as well. Let $p_{j}$ be one such correct process. We have to prove that $p_{j}$ RTBAB-delivers $m$ also at instance inst. By RTBRB-Agreement, it must be that $p_{j}$ eventually receives the same broadcasts as $p_{i}$, among other things, those for which $p_{l}$ is the leader. From RTBC-Agreement and RTBCTermination, it must be that all correct processes eventually decide the same values for each RTBC instance. Therefore, $p_{j}$ will eventually reach instance inst, and will therefore also RTBAB-deliver $m$.

Lemma 23 (variable-dependent RTBAB-Timeliness). There exists a known $\Delta_{\mathrm{A}}$ such that if a correct process $p_{i}$ broadcasts $m$ at time $t$, no correct process delivers $m$ after real time $t+$

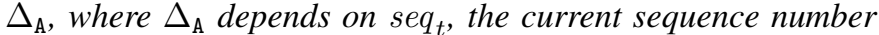
at the time $m$ is broadcasted.

Proof outline. Timeliness follows from RTBRB-Timeliness and RTBC-Timeliness, as well as of the fact that Algorithm 3 rotates through the processes (processes might have to wait a full rotation before they get a chance to decide on a messages that was RTBAB-broadcasted). Let $\Delta_{C}$ be the time it takes for all correct processes to decide on a value using RTBC (see RTBC-Timeliness). Let $\Delta_{R}$ be the time it takes for all correct processes to deliver a message using RTBRB (which exists by RTBRB-Timeliness). Assume that $p_{i}$ assigns the sequence number $s e q_{t}$ with the message $m$. As mentioned above, we assume that $p_{i}$ RTBAB-broadcasts $m$ at time $t$. Because correct processes might still be RTBRB-delivering messages when they gets the RTBRB-deliver message for $m$, they might not be able to RTBRB-deliver $m$ right away (it might be that $\left.s e q_{t}>n e x t\left[p_{i}\right]\right)$. However, we are guaranteed that all correct processes will have delivered $m$ by time $T_{1}=t+\left(\left(\Delta_{R}+\Delta_{\mathrm{W}}\right) \times\left(s e q_{t}+1\right)\right)$ (where $\Delta_{\mathrm{W}}$ is the time processes wait for before re-trying to handle a message-see line 15 and line 36). Note that at that time, processes might be RTBAB-delivering other messages broadcasted by other processes than $p_{i}$. Also, there might already be some messages from $p_{i}$ to RTBAB-deliver before $m$ (all those with sequence numbers less than $s e q_{t}$ ). In case $p_{i}$ is currently not the leader, it might have to wait a full rotation through the processes to get a chance to be the leader again. Given the fact that all correct processes have $m$ in their unordered list by time $T_{1}$, a full rotation will take at most $n \times\left(\Delta_{\mathrm{C}}+\Delta_{\mathrm{W}}\right)$. Because processes might have to process $s e q_{t}$ messages from $p_{i}$ before they get a chance to process $m$, it follows that $m$ will be RTBAB-delivered by $t+\left(\left(\Delta_{\mathrm{R}}+\Delta_{\mathrm{W}}\right) \times\left(s e q_{t}+1\right)\right)+\left(n \times\left(\Delta_{\mathrm{C}}+\Delta_{\mathrm{W}}\right) \times\left(s e q_{t}+1\right)\right)$.

As mentioned in Def. 4, the RTBAB timeliness bound is different from the RTBRB one. $\Delta_{\mathrm{A}}$ is the RTBAB bound, while $\Delta_{R}$ is the RTBRB bound.

Lemma 24 (RTBAB-Timeliness). Under Assumption 3, there exists a known $\Delta_{\mathrm{A}}$ such that if a correct process $p_{i}$ broadcasts $m$ at time $t$, no correct process delivers $m$ after real time $t+$ $\Delta_{\mathrm{A}}$.

Proof outline. Using Assumption 3 and a proof similar to the one of Lemma 23, we derive that messages RTBABbroadcasted at time $t$ are RTBAB-delivered by $t+\left(\Delta_{\mathrm{R}}+\Delta_{\mathrm{W}}+\right.$ $\left.\left(n \times\left(\Delta_{\mathrm{C}}+\Delta_{\mathrm{W}}\right)\right)\right)$.

Lemma 25 (RTBAB-Total order). Let $m_{1}$ and $m_{2}$ be any two messages and suppose that $p_{i}$ and $p_{j}$ are any two correct

As mentioned in Def. 4 , in addition to the RTBRB properties, RTBAB also include a total order property. processes that deliver $m_{1}$ and $m_{2}$. If $p_{i}$ delivers $m_{1}$ before $m_{2}$, then $p_{j}$ delivers $m_{1}$ before $m_{2}$.

Proof outline. Because $p_{i}$ RTBAB-delivers $m_{1}$ before $m_{2}$, it must have RTBC-decided $m_{1}$ at an instance inst $_{1}$ and $m_{2}$ at an instance inst $_{2}$ such that inst $_{1}<$ inst $_{2}$. By RTBC-Agreement and RTBC-Termination, $p_{j}$ must also have RTBC-decided $m_{1}$ at inst $_{1}$ and $m_{2}$ at inst $_{2}$. Using a similar argument as in the proof of RTBAB-Agreement, we derive that $p_{j}$ must then also have RTBAB-delivered $m_{1}$ at instance inst $t_{1}$ and $m_{2}$ at inst $_{2}$.

\section{APPENDIX E \\ Evaluation Using Number of Messages SENT}

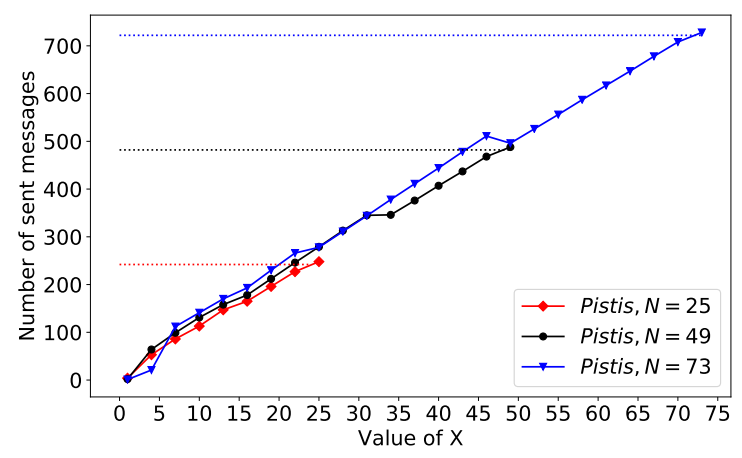

Figure 12. Average number of messages transmitted per node with a $1 \mathrm{~ms}$ link latency, with system sizes equal to 25, 49 and 73 for Pistis and RT-ByzCast.

To complement the bandwidth consumption evaluation that was previously reported, Fig. 12 presents the number of messages transmitted using either Pistis or RT-ByzCast. We considered systems containing 25, 49 and 73 nodes (i.e., $3 \mathrm{f}+1$ for $\mathrm{f}$ equals to 8,16 and 24 ). We used a $1 \mathrm{~ms}$ network latency and 1B messages. RT-ByzCast's values are reported with dashed horizontal lines. One can see that Pistis sends less messages when the value of $X$ decreases. In addition, PISTIS always sends less messages than RT-ByzCast. In particular, PISTIS and RT-ByzCast approximately send the same number of messages when $X=3 f+1$. These results are consistent with the bandwidth consumption results reported in Sec. VI-E, and which therefore indicate that the main reason behind Pistis' lower bandwidth consumption is a smaller number of messages exchanged.

\section{References}

[1] D. Dolev, C. Dwork, and L. Stockmeyer. "On the Minimal Synchronism Needed for Distributed Consensus". In: JACM 34.1 (1987).

[2] D. Dzung, R. Guerraoui, D. Kozhaya, and Y.-A. Pignolet. "Never Say Never - Probabilistic and Temporal Failure Detectors". In: IPDPS. 2016.

[3] T. D. Chandra and S. Toueg. "Unreliable Failure Detectors for Reliable Distributed Systems". In: JACM 43.2 (1996), pp. 225-267.

[4] P. Verissimo and C. Almeida. "Quasi-Synchronism: a step away from the traditional fault-tolerant real-time system models". In: Bulletin of the Technical Committee on Operating Systems and Application Environments (TCOS) 7.4 (1995), pp. 35-39.

[5] M. Pease, R. Shostak, and L. Lamport. "Reaching Agreement in the Presence of Faults". In: JACM 27.2 (1980), pp. 228-234. 